\title{
Strong calmness of perturbed KKT system for a class of conic programming with degenerate solutions *
}

\author{
Yulan Liu ${ }^{\dagger}$ and Shaohua Pan ${ }^{\ddagger}$
}

October 10, 2018

\begin{abstract}
This paper is concerned with the strong calmness of the KKT solution mapping for a class of canonically perturbed conic programming, which plays a central role in achieving fast convergence under situations when the Lagrange multiplier associated to a solution of these conic optimization problems is not unique. We show that the strong calmness of the KKT solution mapping is equivalent to a local error bound for solutions of perturbed KKT system, and is also equivalent to the pseudo-isolated calmness of the stationary point mapping along with the calmness of the multiplier set map at the corresponding reference point. Sufficient conditions are also provided for the strong calmness by establishing the pseudo-isolated calmness of the stationary point mapping in terms of the noncriticality of the associated multiplier, and the calmness of the multiplier set mapping in terms of a relative interior condition for the multiplier set. These results cover and extend the existing ones in $[16,20]$ for nonlinear programming and in $[7,35]$ for semidefinite programming.
\end{abstract}

Keywords: KKT solution mapping; strong calmness; local error bound; pseudo-isolated calmness; noncritical multiplier

Mathematics Subject Classification(2010): 49K40, 90C31, 49J53

\section{Introduction}

Let $\mathbb{X}$ and $\mathbb{Y}$ be two finite dimensional real vector spaces equipped with an inner product $\langle\cdot, \cdot\rangle$ and its induced norm $\|\cdot\|$. Let $f: \mathbb{X} \rightarrow \mathbb{R}$ and $g: \mathbb{X} \rightarrow \mathbb{Y}$ be twice continuously differentiable functions. We consider the canonically perturbed optimization problem

$$
\min _{x \in \mathbb{X}}\{f(x)-\langle a, x\rangle: g(x)-b \in \mathcal{K}\},
$$

${ }^{*}$ Supported by the National Natural Science Foundation of China under project No.11571120 and the Natural Science Foundation of Guangdong Province under project No.2015A030313214.

${ }^{\dagger}$ School of Applied Mathematics, Guangdong University of Technology(ylliu@gdut.edu.cn.

${ }^{\ddagger}$ School of Mathematics, South China University of Technology, Guangzhou (shhpan@scut.edu.cn). 
where $(a, b) \in \mathbb{X} \times \mathbb{Y}$ is the perturbation parameter and $\mathcal{K} \subseteq \mathbb{Y}$ is a nonempty closed convex set. Throughout this paper, we assume that $\mathcal{K}$ is $C^{2}$-cone reducible (see Definition 2.4), which covers all polyhedral convex sets and several classes of important non-polyhedral convex cones such as the positive semidefinite cone [4, Corollary 4.6], the second-order cone [5, Lemma 15] and the epigraph cone of the Ky Fan matrix $k$-norm [8].

Let $L: \mathbb{X} \times \mathbb{Y} \rightarrow \mathbb{R}$ be the Lagrange function of problem (1) without perturbation:

$$
L(x, \lambda):=f(x)+\langle\lambda, g(x)\rangle \quad \forall(x, \lambda) \in \mathbb{X} \times \mathbb{Y} .
$$

For a given perturbation $(a, b)$, the KKT optimality condition for (1) takes the form of

$$
\left\{\begin{array} { l } 
{ \nabla _ { x } L ( x , \lambda ) = a ; } \\
{ \lambda \in \mathcal { N } _ { \mathcal { K } } ( g ( x ) - b ) }
\end{array} \Longleftrightarrow \left\{\begin{array}{l}
\nabla f(x)+\nabla g(x) \lambda=a ; \\
g(x)-b=\Pi_{\mathcal{K}}(g(x)-b+\lambda)
\end{array}\right.\right.
$$

where $\mathcal{N}_{\mathcal{K}}(z)$ denotes the normal cone of $\mathcal{K}$ at $z$ in the sense of convex analysis [29], $\Pi_{\mathcal{K}}$ means the projection operator onto $\mathcal{K}$, and for any given $\lambda \in \mathbb{Y}, \nabla_{x} L(\cdot, \lambda)$ is the adjoint of $L_{x}^{\prime}(\cdot, \lambda)$, the derivative of $L(\cdot, \lambda)$ at $x \in \mathbb{X}$. In this paper, for a twice continuously differentiable $h: \mathbb{X} \rightarrow \mathbb{Y}$, we denote by $h^{\prime}(x)$ the first-order derivative of $h$ at $x$, by $\nabla h(x)$ the adjoint of $h^{\prime}(x)$, and by $h^{\prime \prime}(x)$ the second-order derivative of $h$ at $x$. We define the KKT solution mapping $\mathcal{S}_{\mathrm{KKT}}: \mathbb{X} \times \mathbb{Y} \rightrightarrows \mathbb{X} \times \mathbb{Y}$, the stationary point map $\mathcal{X}_{\mathrm{KKT}}: \mathbb{X} \times \mathbb{Y} \rightrightarrows \mathbb{X}$, and the multiplier set map $\mathcal{M}: \mathbb{X} \times \mathbb{X} \times \mathbb{Y} \rightrightarrows \mathbb{Y}$ respectively by

$$
\begin{aligned}
& \mathcal{S}_{\mathrm{KKT}}(a, b):=\left\{(x, \lambda) \in \mathbb{X} \times \mathbb{Y} \mid \nabla_{x} L(x, \lambda)=a, \lambda \in \mathcal{N}_{\mathcal{K}}(g(x)-b)\right\}, \\
& \mathcal{X}_{\mathrm{KKT}}(a, b):=\{x \in \mathbb{X} \mid \exists \lambda \in \mathbb{Y} \text { such that system }(2) \text { holds at }(x, \lambda)\}, \\
& \mathcal{M}(x, a, b):=\left\{\lambda \in \mathbb{Y} \mid(x, \lambda) \in \mathcal{S}_{\mathrm{KKT}}(a, b)\right\} .
\end{aligned}
$$

In addition, in the sequel we also need a counterpart of the multifunction $\mathcal{M}$, defined by

$$
\mathcal{X}(\lambda, a, b):=\left\{x \in \mathbb{X} \mid(x, \lambda) \in \mathcal{S}_{\mathrm{KKT}}(a, b)\right\} .
$$

Clearly, the multifunction $\mathcal{X}$ can be regarded as a localization version of $\mathcal{X}_{\mathrm{KKT}}$.

This work is mainly concerned with the strong calmness of $\mathcal{S}_{\mathrm{KKT}}$ at a reference point $(\bar{x}, \bar{\lambda}) \in \mathcal{S}_{\mathrm{KKT}}(0,0)$ with $\mathcal{M}(\bar{x}, 0,0) \neq\{\bar{\lambda}\}$, that is, $\bar{x}$ is a degenerate stationary point of the problem $(1)$ with $(a, b)=(0,0)$. The strong calmness is formally defined as follows.

Definition 1.1 Let $(\bar{x}, \bar{\lambda})$ be a KKT point of the problem $(1)$ with $(a, b)=(0,0)$ and $\mathcal{M}(\bar{x}, 0,0) \neq\{\bar{\lambda}\}$. The multifunction $\mathcal{S}_{\mathrm{KKT}}$ is said to have the strong calmness at the origin for $(\bar{x}, \bar{\lambda})$ if there exist $\delta>0, \varepsilon>0$ and a constant $\kappa>0$ such that for any $(a, b) \in \mathbb{B}_{\delta}((0,0))$ and any $(x, \lambda) \in \mathcal{S}_{\mathrm{KKT}}(a, b) \cap \mathbb{B}_{\varepsilon}((\bar{x}, \bar{\lambda}))$, the following estimate holds:

$$
\|x-\bar{x}\|+\operatorname{dist}(\lambda, \mathcal{M}(\bar{x}, 0,0)) \leq \kappa\|(a, b)\| .
$$

This property, different from the locally upper Lipschitz introduced by Robinson [30] for a multifunction, is weaker than the isolated calmness of $\mathcal{S}_{\mathrm{KKT}}$ but stronger than its calmness. Moreover, it does not imply the isolatedness of the stationary point $\bar{x}$. For 
the definition of (isolated) calmness, the reader may refer to [10] or Section 2. When $\mathcal{M}(\bar{x}, 0,0)=\{\bar{\lambda}\}$, this property becomes the isolated calmness of $\mathcal{S}_{\mathrm{KKT}}$ at the origin.

To the best of our knowledge, the strong calmness in Definition 1.1 was first introduced by Fernández and Solodov [11] to guarantee the calmness of the KKT solution mapping for the canonically perturbed nonlinear programming, and then obtain the superlinear convergence of the stabilized sequential quadratic programming method (sSQP) by invoking [12, Theorem 1]. Later, Izmailov and Solodov [20, 21] provided some equivalent characterizations for the strong calmness of the KKT solution mapping in the setting of polyhedral conic optimization. They showed that this upper Lipschitz stability is not only equivalent to a local error bound for solutions of perturbed KKT system, but also equivalent to the noncriticality of the associated multiplier. As discussed in $[33,16,11,20,21,22]$, the strong calmness of the KKT solution map or equivalently the noncriticality of the associated multiplier is the key to achieve a fast convergence rate for the SSQP method or the augmented Lagrangian method (ALM) of the polyhedral conic optimization problems with degenerate solutions, i.e., the solutions with multiple Lagrange multipliers. Then, it is natural to ask whether these characterizations hold or not for nonpolyhedral conic optimization. If not, what conditions are enough?

The main contribution of this work is to provide an affirmative answer to this question. Specifically, in Section 3 we show that the strong calmness of $\mathcal{S}_{\mathrm{KKT}}$ at the origin for $(\bar{x}, \bar{\lambda}) \in \mathcal{S}_{\mathrm{KKT}}(0,0)$ is equivalent to the local error bound stated in Property 1 for solutions of perturbed KKT system, and is also equivalent to the pseudo-isolated calmness (see Definition 3.1) of the stationary point map $\mathcal{X}_{\mathrm{KKT}}$ at the origin for $\bar{x}$ together with the calmness of the multiplier set map $\mathcal{M}$ at $(\bar{x}, 0,0)$ for $\bar{\lambda}$. Among others, the calmness of $\mathcal{M}$ at $(\bar{x}, 0,0)$ for $\bar{\lambda}$ is very weak and holds automatically in the polyhedral setting. However, unlike the polyhedral case, the noncriticality of the Lagrange multiplier $\bar{\lambda}$ is only necessary but not sufficient for the pseudo-isolated calmness of $\mathcal{X}_{\text {KKT }}$. In Section 4, we show that, under some restrictions on the mapping $\nabla g(\bar{x}): \mathbb{X} \rightarrow \mathbb{Y}$, the noncriticality of the multiplier $\bar{\lambda}$ can guarantee the pseudo-isolated calmness of $\mathcal{X}_{\mathrm{KKT}}$, and consequently, some sufficient characterizations for the strong calmness of $\mathcal{S}_{\mathrm{KKT}}$ are obtained.

Notice that for structured convex semidefinite programming with multiple solutions (actually degenerate solutions), Cui, Sun and Toh [7] have studied the calmness of the stationary point mapping and the strong calmness of the perturbed KKT system so as to capture the fast convergence of the ALM for such problems. Recently, for general nonlinear semidefinite programming, Zhang and Zhang [35] established the strong calmness of $\mathcal{S}_{\mathrm{KKT}}$ under the noncriticality of the Lagrange multiplier along with some additional conditions, which improves the result in [7] for the perturbed KKT system since the noncriticality of the multiplier is much weaker than the second-order sufficient condition in [7] associated to a specified multiplier. This paper is to a great extent motivated by their works. We extend their results to a class of conic programming and remove the condition (i) of [35, Theorem 3.3] under the relative interior condition of multiplier set.

In the rest of this paper, for a finite dimensional vector space $\mathbb{Z}$ equipped with a norm $\|\cdot\|, \mathbb{B}_{\mathbb{Z}}$ denotes the closed unit ball centered at the origin in $\mathbb{Z}$ and for a given $z \in \mathbb{Z}$, 
$\mathbb{B}_{\delta}(z)$ means the closed ball of radius $\delta$ centered at $z$. For a given closed set $\Omega \subseteq \mathbb{Z}, \Pi_{\Omega}(\cdot)$ denotes the projection operator onto $\Omega$, and $\operatorname{dist}(x, \Omega):=\inf _{z \in \Omega}\|z-x\|$ for a given $x \in \mathbb{Z}$ means the distance of $x$ from the set $\Omega$; and for a given nonempty convex cone $K \subseteq \mathbb{Y}$, $K^{\circ}$ denotes the negative polar of $K$. For a linear map $\mathcal{A}, \mathcal{A}^{*}$ denotes the adjoint of $\mathcal{A}$.

\section{Preliminaries}

Throughout this section, $\mathbb{Z}$ and $\mathbb{W}$ denote two finite dimensional vector spaces equipped with the inner product $\langle\cdot, \cdot\rangle$ and its induced norm $\|\cdot\|$. We first recall some background knowledge from the excellent monographs $[32,2,23,28,10]$. Let $\Omega \subseteq \mathbb{Z}$ be a nonempty set. Fix an arbitrary $\bar{z} \in \Omega$. The contingent cone to $\Omega$ at $\bar{z}$ is defined as

$$
\mathcal{T}_{\Omega}(\bar{z}):=\left\{w \in \mathbb{Z} \mid \exists t_{k} \downarrow 0, w^{k} \rightarrow w \text { as } k \rightarrow \infty \text { with } \bar{x}+t_{k} w_{k} \in \Omega\right\},
$$

while the basic/limiting normal cone to $\Omega$ at $\bar{z}$ admits the following representation

$$
\mathcal{N}_{\Omega}(\bar{z})=\limsup _{z \underset{\Omega}{\rightarrow}} \widehat{\mathcal{N}}_{\Omega}(z) \text { with } \widehat{\mathcal{N}}_{\Omega}(z):=\left\{v \in \mathbb{Z} \mid \limsup _{z \underset{\Omega}{\mathrm{z}}} \frac{\langle v, z-\bar{z}\rangle}{\|z-\bar{z}\|} \leq 0\right\} .
$$

When $\Omega$ is locally closed around $\bar{z} \in \Omega$, this is equivalent to the original definition by Mordukhovich [27], i.e., $\mathcal{N}_{\Omega}(\bar{z}):=\lim \sup _{z \rightarrow \bar{z}}\left[\operatorname{cone}\left(z-\Pi_{\Omega}(z)\right)\right]$, and when $\Omega$ is convex, $\mathcal{N}_{\Omega}(\bar{z})$ becomes the normal cone in the sense of convex analysis [29].

Let $\mathcal{F}: \mathbb{Z} \rightrightarrows \mathbb{W}$ be a given multifunction. Consider an arbitrary $(\bar{z}, \bar{w}) \in \operatorname{gph} \mathcal{F}$ such that $\mathcal{F}$ is locally closed at $(\bar{z}, \bar{w})$, where gph $\mathcal{F}$ denotes the graph of $\mathcal{F}$. We recall from $[32,10]$ the concepts of metric subregularity and calmness of the multifunction $\mathcal{F}$.

Definition 2.1 The multifunction $\mathcal{F}: \mathbb{Z} \rightrightarrows \mathbb{W}$ is said to be metrically subregular at $\bar{z}$ for $\bar{w} \in \mathcal{F}(\bar{z})$ if there exists $\kappa>0$ along with $\varepsilon>0$ and $\delta>0$ such that for all $z \in \mathbb{B}_{\varepsilon}(\bar{z})$,

$$
\operatorname{dist}\left(z, \mathcal{F}^{-1}(\bar{w})\right) \leq \kappa \operatorname{dist}\left(\bar{w}, \mathcal{F}(z) \cap \mathbb{B}_{\delta}(\bar{w})\right) .
$$

Definition 2.2 The multifunction $\mathcal{F}$ is said to be calm at $\bar{z}$ for $\bar{w}$ if there exists $\kappa>0$ along with $\varepsilon>0$ and $\delta>0$ such that for all $z \in \mathbb{B}_{\varepsilon}(\bar{z})$,

$$
\mathcal{F}(z) \cap \mathbb{B}_{\delta}(\bar{w}) \subseteq \mathcal{F}(\bar{z})+\kappa\|z-\bar{z}\| \mathbb{B}_{\mathbb{W}} .
$$

If in addition $\mathcal{F}(\bar{z})=\{\bar{w}\}$, the multifunction $\mathcal{F}$ is said to be isolated calm at $\bar{z}$ for $\bar{w}$.

By [10, Exercise 3H.4], the neighborhood $\mathbb{B}_{\delta}(\bar{w})$ in Definition 2.1 and the restriction of $z \in \mathbb{B}_{\varepsilon}(\bar{z})$ in Definition 2.2 can be removed. The graphical derivative of $\mathcal{F}$ is a convenient tool to study the isolated calmness of $\mathcal{F}$. Recall from [1] the graphical derivative of $\mathcal{F}$ at $(\bar{z}, \bar{w})$ is the mapping $D \mathcal{F}(\bar{z} \mid \bar{w}): \mathbb{Z} \rightrightarrows \mathbb{W}$ defined by $\Delta w \in D \mathcal{F}(\bar{z} \mid \bar{w})(\Delta z)$ if and only if $(\Delta z, \Delta w) \in \mathcal{T}_{\operatorname{gph} \mathcal{F}}(\bar{z}, \bar{w})$. With the graphical derivative of $\mathcal{F}$, the following result holds.

Lemma 2.1 (see [24, Proposition 2.1] or [26, Proposition 4.1]) The multifunction $\mathcal{F}$ is isolated calm at $\bar{z}$ for $\bar{w}$ if and only if $D \mathcal{F}(\bar{z} \mid \bar{w})(0)=\{0\}$. 
Let $\bar{x}$ be a feasible point of the problem $(1)$ with $(a, b)=(0,0)$. The critical cone of (1) with $(a, b)=(0,0)$ at $\bar{x}$ is defined as $\mathcal{C}(\bar{x}):=\left\{d \in \mathbb{X} \mid g^{\prime}(\bar{x}) d \in \mathcal{T}_{\mathcal{K}}(g(\bar{x})),\langle\nabla f(\bar{x}), d\rangle \leq 0\right\}$. When $\bar{x}$ is a stationary point and $\bar{\lambda} \in \mathcal{M}(\bar{x}, 0,0)$, the critical cone can be rewritten as

$$
\mathcal{C}(\bar{x})=\left\{d \in \mathbb{X} \mid g^{\prime}(\bar{x}) d \in \mathcal{C}_{\mathcal{K}}(g(\bar{x}), \bar{\lambda})\right\}
$$

where, for any $y \in \mathcal{K}, \mathcal{C}_{\mathcal{K}}(y, u)$ is the critical cone of $\mathcal{K}$ at $y$ w.r.t. $u \in \mathcal{N}_{\mathcal{K}}(y)$, defined by

$$
\mathcal{C}_{\mathcal{K}}(y, u):=\mathcal{T}_{\mathcal{K}}(y) \cap u^{\perp} .
$$

Motivated by [35, Definition 3.2], we introduce the concept of noncritical multipliers.

Definition 2.3 Let $\bar{x}$ be a stationary point of $(1)$ with $(a, b)=(0,0)$ and $\bar{\lambda} \in \mathcal{M}(\bar{x}, 0,0)$. The Lagrange multiplier $\bar{\lambda}$ is said to be noncritical if the following generalized equation

$$
\left.0 \in \nabla_{x x}^{2} L(\bar{x}, \bar{\lambda}) \xi+\nabla g(\bar{x}) D \mathcal{N}_{\mathcal{K}}(g(\bar{x}) \mid \bar{\lambda})\right)\left(g^{\prime}(\bar{x}) \xi\right)
$$

has the unique trivial solution $\xi=0$, and otherwise $\bar{\lambda}$ is said to be critical.

Now let us recall the definition of the $C^{2}$-cone reducibility for a closed convex set.

Definition 2.4 ([2, Definition 3.135]) A closed convex set $\Omega$ in $\mathbb{Y}$ is said to be $C^{2}$-cone reducible at $\bar{y} \in \Omega$, if there exist an open neighborhood $\mathcal{Y}$ of $\bar{y}$, a pointed closed convex cone $D \subseteq \mathbb{Z}$ and a twice continuously differentiable mapping $\Xi: \mathcal{Y} \rightarrow \mathbb{Z}$ such that (i) $\Xi(\bar{y})=0$; (ii) $\Xi^{\prime}(\bar{y}): \mathbb{Y} \rightarrow \mathbb{Z}$ is onto; (iii) $\Omega \cap \mathcal{Y}=\{y \in \mathcal{Y} \mid \Xi(y) \in D\}$. We say that the closed convex set $\Omega$ is $C^{2}$-cone reducible if $\Omega$ is $C^{2}$-cone reducible at every $y \in \Omega$.

Since $\mathcal{K}$ is assumed to be $C^{2}$-cone reducible in this paper, by [2, Proposition 3.136] the set $\mathcal{K}$ is second-order regular at each $y \in \mathcal{K}$, and hence $\mathcal{T}_{\mathcal{K}}^{i, 2}(y, h)=\mathcal{T}_{\mathcal{K}}^{2}(y, h)$ at each $y \in \mathcal{K}$ for any $h \in \mathbb{Y}$, where $\mathcal{T}_{\mathcal{K}}^{i, 2}(y, h)$ and $\mathcal{T}_{\mathcal{K}}^{2}(y, h)$ denotes the inner and outer second order tangent sets to $\mathcal{K}$ at $y$ in the direction $h \in \mathbb{Y}$. From the standard reduction approach, we have the following result on the representation of the normal cone of $\mathcal{K}$ and the "sigma term" of the $C^{2}$-cone reducible set $\mathcal{K}$ (see [2, Equation(3.266)\&(3.274)]).

Lemma 2.2 Let $\bar{y} \in \mathcal{K}$ be given. There exist an open neighborhood $\mathcal{Y}$ of $\bar{y}$, a pointed closed convex cone $D \subseteq \mathbb{Z}$, and a twice continuously differentiable mapping $\Xi: \mathcal{Y} \rightarrow \mathbb{Z}$ satisfying conditions (i)-(iii) in Definition 2.4 such that for all $y \in \mathcal{Y}$,

$$
\mathcal{N}_{\mathcal{K}}(y)=\nabla \Xi(y) \mathcal{N}_{D}(\Xi(y)) .
$$

Also, for any $\bar{\lambda} \in \mathcal{N}_{\mathcal{K}}(\bar{y})$, there exists a unique $\bar{\mu} \in \mathcal{N}_{D}(\Xi(\bar{y}))$ such that $\bar{\lambda}=\nabla \Xi(\bar{y}) \bar{\mu}$, and

$$
\Upsilon(h):=-\sigma\left(\bar{\lambda}, \mathcal{T}_{\mathcal{K}}^{2}(\bar{y}, h)\right)=\left\langle\bar{\mu}, \Xi^{\prime \prime}(\bar{y})(h, h)\right\rangle \quad \forall h \in \mathcal{C}_{\mathcal{K}}(\bar{y}, \bar{\lambda}) .
$$

Next we recall a useful result on the directional derivative of the projection operator $\Pi_{\mathcal{K}}$. Fix an arbitrary $y \in \mathbb{Y}$. Write $\bar{y}=\Pi_{\mathcal{K}}(y)$ and let $\bar{\lambda} \in \mathcal{N}_{\mathcal{K}}(\bar{y})$. Since $\mathcal{K}$ is $C^{2}$-cone 
reducible, by [4, Theorem 7.2$]$ the mapping $\Pi_{\mathcal{K}}$ is directionally differentiable at $y$ and the directional derivative $\Pi_{\mathcal{K}}^{\prime}(y ; h)$ for any direction $h \in \mathbb{Y}$ satisfies

$$
\Pi_{\mathcal{K}}^{\prime}(y ; h)=\underset{d \in \mathbb{Y}}{\arg \min }\left\{\|d-h\|^{2}-\sigma\left(\bar{\lambda}, \mathcal{T}_{\mathcal{K}}^{2}(\bar{y}, d)\right): d \in \mathcal{C}_{\mathcal{K}}(\bar{y}, \bar{\lambda})\right\}
$$

In addition, by following the arguments as those for [34, Theorem 3.1], one can obtain

$$
\mathcal{T}_{\mathrm{gph} \mathcal{N}_{\mathcal{K}}}(z, w)=\left\{(\Delta z, \Delta w) \in \mathbb{Y} \times \mathbb{Y} \mid \Pi_{\mathcal{K}}^{\prime}(z+w ; \Delta z+\Delta w)=\Delta z\right\} .
$$

Combining this with [9, Lemma 10], we have the following conclusion for the graphical derivative of $\mathcal{N}_{\mathcal{K}}$, the directional derivative of $\Pi_{\mathcal{K}}$ and the critical cone of the set $\mathcal{K}$.

Lemma 2.3 Let $z \in \mathbb{Y}$ be a given vector. Write $\bar{z}:=\Pi_{\mathcal{K}}(z)$ and $\bar{\mu}:=z-\bar{z}$. Then, with $\Upsilon(\cdot)=-\sigma\left(\bar{\mu}, \mathcal{T}_{\mathcal{K}}^{2}(y, \cdot)\right)=\left\langle u, \Xi^{\prime \prime}(\bar{z})(\cdot, \cdot)\right\rangle$ for $u \in \mathcal{N}_{D}(\Xi(\bar{z}))$, it holds that

$$
\begin{aligned}
\Delta \lambda \in D \mathcal{N}_{\mathcal{K}}(\bar{z} \mid \bar{\mu})(\Delta y) & \Longleftrightarrow \Delta y-\Pi_{\mathcal{K}}^{\prime}(z ; \Delta y+\Delta \lambda)=0 \\
& \Longleftrightarrow\left\{\begin{array}{l}
\Delta y \in \mathcal{C}_{\mathcal{K}}(\bar{z}, \bar{\mu}), \\
\Delta \lambda-\frac{1}{2} \nabla \Upsilon(\Delta y) \in\left[\mathcal{C}_{\mathcal{K}}(\bar{z}, \bar{\mu})\right]^{\circ}, \\
\langle\Delta y, \Delta \lambda\rangle=-\sigma\left(\bar{\mu}, \mathcal{T}_{\mathcal{K}}^{2}(\bar{z}, \Delta y)\right) .
\end{array}\right.
\end{aligned}
$$

By using Lemma 2.3 and Definition 2.3, it is immediate to obtain the following result.

Proposition 2.1 Let $(\bar{x}, \bar{\lambda})$ be a KKT point of the problem $(1)$ with $(a, b)=(0,0)$. Then $\bar{\lambda}$ is noncritical iff all solutions of the following system have the form of $(0, *) \in \mathbb{X} \times \mathbb{Y}$ :

$$
\left\{\begin{array}{l}
\nabla_{x x}^{2} L(\bar{x}, \bar{\lambda}) \xi+\nabla g(\bar{x}) v=0 \\
g^{\prime}(\bar{x}) \xi-\Pi_{\mathcal{K}}^{\prime}\left(g(\bar{x})+\bar{\lambda} ; g^{\prime}(\bar{x}) \xi+v\right)=0
\end{array}\right.
$$

To close this section, we study the calmness of a multifunction related to the perturbed KKT system. For a given $x \in \mathbb{X}$, we define the multifunction $\mathcal{G}_{x}: \mathbb{X} \times \mathbb{Y} \rightrightarrows \mathbb{Y}$ by

$$
\mathcal{G}_{x}(\eta, y):=\left\{\lambda \in \mathbb{Y} \mid \eta+\nabla g(x) \lambda=0, y-\Pi_{\mathcal{K}}(y+\lambda)=0\right\} .
$$

Let $(\bar{x}, \bar{\lambda})$ be a KKT point of $(1)$ with $(a, b)=(0,0)$. Clearly $\mathcal{G}_{\bar{x}}(\nabla f(\bar{x}), g(\bar{x}))=\mathcal{M}(\bar{\lambda}, 0,0)$. The following proposition gives some conditions for the calmness of $\mathcal{G}_{\bar{x}}$ at $(\nabla f(\bar{x}), g(\bar{x}))$ for $\bar{\lambda}$ which, as will be seen in Section 3, are sufficient for that of $\mathcal{M}$ at $(\bar{x}, 0,0)$ for $\bar{\lambda}$.

Proposition 2.2 Let $(\bar{x}, \bar{\lambda})$ be a KKT point of the problem $(1)$ with $(a, b)=(0,0)$. Write $\bar{\eta}:=\nabla f(\bar{x})$ and $\bar{y}:=g(\bar{x})$. Define the multifunction $\mathcal{E}(\eta):=\mathcal{H}(\eta) \cap \mathcal{N}_{\mathcal{K}}(\bar{y})$ for $\eta \in \mathbb{X}$, where $\mathcal{H}(\eta):=\{\lambda \in \mathbb{Y} \mid \eta+\nabla g(\bar{x}) \lambda=0\}$. Then the following statements hold:

(a) the multifunction $\mathcal{G}_{\bar{x}}$ is calm at $(\bar{\eta}, \bar{y})$ for $\bar{\lambda}$ provided that $\mathcal{E}$ is calm at $\bar{\eta}$ for $\bar{\lambda}$;

(b) $\mathcal{E}$ is calm at $\bar{\eta}$ for $\bar{\lambda}$ if there exist $\delta>0$ and $\gamma>0$ such that for any $\lambda \in \mathbb{B}_{\delta}(\bar{\lambda})$,

$$
\operatorname{dist}(\lambda, \mathcal{E}(\bar{\eta})) \leq \gamma \max \left\{\operatorname{dist}\left(\lambda, \mathcal{N}_{\mathcal{K}}(\bar{y})\right), \operatorname{dist}(\lambda, \mathcal{H}(\bar{\eta}))\right\},
$$

which is particularly implied by the condition that $\operatorname{ri}\left(\mathcal{N}_{\mathcal{K}}(\bar{y})\right) \cap \mathcal{H}(\bar{\eta}) \neq \emptyset$. 
Proof: (a) Notice that $\mathcal{G}_{\bar{x}}(\eta, y)=\mathcal{H}(\eta) \cap \mathcal{N}_{\mathcal{K}}(y)$ for any $(\eta, y) \in \mathbb{X} \times \mathbb{Y}$. Since $\mathcal{K}$ is assumed to be $C^{2}$-cone reducible, the multifunction $\mathcal{N}_{\mathcal{K}}$ is calm at $\bar{y}$ for $\bar{\lambda}$ by $[25$, Theorem 2.1]. Since the multifunction $\mathcal{H}$ is polyhedral, from [30, Proposition 1] we know that $\mathcal{H}$ is calm at $\bar{\eta}$ for $\bar{\lambda}$. By [23, Theorem 3.6] and the given assumption that $\mathcal{E}$ is calm at $\bar{\eta}$ for $\bar{\lambda}$, it suffices to check that $\mathcal{H}^{-1}$ has the Aubin property at $\bar{\lambda}$ for $\bar{\eta}$. For this purpose, fix arbitrary $\lambda, \lambda^{\prime} \in \mathbb{Y}$ and take an arbitrary $u \in \mathcal{H}^{-1}(\lambda)$. Then, we have $u+\nabla g(\bar{x}) \lambda=0$. Notice that $\mathcal{H}^{-1}\left(\lambda^{\prime}\right)$ is a closed convex set. Let $u^{\prime} \in \mathcal{H}^{-1}\left(\lambda^{\prime}\right)$ be such that $\left\|u-u^{\prime}\right\|=\operatorname{dist}\left(u, \mathcal{H}^{-1}\left(\lambda^{\prime}\right)\right)$. Then, by using $u^{\prime}+\nabla g(\bar{x}) \lambda^{\prime}=0$, it holds that

$$
\operatorname{dist}\left(u, \mathcal{H}^{-1}\left(\lambda^{\prime}\right)\right)=\left\|u-u^{\prime}\right\|=\left\|-\nabla g(\bar{x}) \lambda+\nabla g(\bar{x}) \lambda^{\prime}\right\| \leq\|\nabla g(\bar{x})\|\left\|\lambda-\lambda^{\prime}\right\| .
$$

This shows that $\mathcal{H}^{-1}$ is Lispchitz continuous in $\mathbb{X}$ by [32, Definition 9.26], and then has the Aubin property at $\bar{\lambda}$ for $\bar{\eta}$. The desired result (a) then follows.

(b) Assume that there exist $\delta>0$ and $\gamma>0$ such that for any $\lambda \in \mathbb{B}_{\delta}(\bar{\lambda})$, inequality (12) holds. Since $\mathcal{H}$ is calm at $\bar{\eta}$ for $\bar{\lambda}$, there exist $\widetilde{\delta}>0$ and $\widetilde{\kappa}>0$ such that

$$
\operatorname{dist}(\lambda, \mathcal{H}(\bar{\eta})) \leq \widetilde{\kappa} \operatorname{dist}\left(\bar{\eta}, \mathcal{H}^{-1}(\lambda)\right) \quad \forall \lambda \in \mathbb{B}_{\widetilde{\delta}}(\bar{\lambda}) .
$$

Set $\delta^{\prime}=\min (\delta, \widetilde{\delta})$. Then, together with inequality (12), for any $\lambda \in \mathbb{B}_{\delta^{\prime}}(\bar{\lambda})$ we have

$$
\operatorname{dist}(\lambda, \mathcal{E}(\bar{\eta})) \leq \gamma \max (1, \widetilde{\kappa}) \max \left\{\operatorname{dist}\left(\lambda, \mathcal{N}_{\mathcal{K}}(\bar{y})\right), \operatorname{dist}\left(\bar{\eta}, \mathcal{H}^{-1}(\lambda)\right)\right\},
$$

which implies that the following inequality holds

$$
\operatorname{dist}(\lambda, \mathcal{E}(\bar{\eta})) \leq \gamma \max (1, \widetilde{\kappa}) \operatorname{dist}\left(\bar{\eta}, \mathcal{H}^{-1}(\lambda)\right) \quad \forall \lambda \in \mathcal{N}_{\mathcal{K}}(\bar{y}) \cap \mathbb{B}_{\delta^{\prime}}(\bar{\lambda}) .
$$

Notice that $\mathcal{E}$ is calm at $\bar{\eta}$ for $\bar{\lambda}$ if and only if $\mathcal{E}^{-1}$ is metrically subregular at $\bar{\lambda}$ for $\bar{\eta}$, which is equivalent to requiring that there exist $\varepsilon>0$ and $\nu>0$ such that

$$
\operatorname{dist}(\lambda, \mathcal{E}(\bar{\eta})) \leq \nu \operatorname{dist}\left(\bar{\eta}, \mathcal{H}^{-1}(\lambda)\right) \quad \forall \lambda \in \mathcal{N}_{\mathcal{K}}(\bar{y}) \cap \mathbb{B}_{\varepsilon}(\bar{\lambda}) .
$$

This shows that the condition in (12) implies the calmness of $\mathcal{E}$ at $\bar{\eta}$ for $\bar{\lambda}$. While the condition in (12) is implied by $\operatorname{ri}\left(\mathcal{N}_{\mathcal{K}}(\bar{y})\right) \cap \mathcal{H}(\bar{\eta}) \neq \emptyset$ by [6, Corollary 3].

Remark 2.1 From [18, Section 3.1] the assumption in (12) is actually a metric qualifcation which is equivalent to the calmness of the following mapping at $(0,0)$ for $\bar{\lambda}$ :

$$
\mathcal{F}(u, v):=\left\{\lambda \in \mathbb{Y}: \lambda+u \in \mathcal{N}_{\mathcal{K}}(\bar{y}), \lambda+v \in \mathcal{H}(\bar{\eta})\right\} .
$$

Clearly, the metric qualification in (12) is weaker than the bounded linear regularity of the collection $\left\{\mathcal{N}_{\mathcal{K}}(\bar{y}), \mathcal{H}(\bar{\eta})\right\}$, while the latter is implied by $\operatorname{ri}\left(\mathcal{N}_{\mathcal{K}}(\bar{y})\right) \cap \mathcal{H}(\bar{\eta}) \neq \emptyset$. 


\section{Equivalent characterizations}

In this section we shall provide two equivalent characterizations for the strong calmness of $\mathcal{S}_{\mathrm{KKT}}$. First of all, we show that the strong calmness of $\mathcal{S}_{\mathrm{KKT}}$ at the origin for $(\bar{x}, \bar{\lambda}) \in \mathcal{S}_{\mathrm{KKT}}(0,0)$ is equivalent to the following error bound for the KKT system.

Property 1 (Error bound for KKT system) Let $(\bar{x}, \bar{\lambda})$ be a KKT point of (1) with $(a, b)=(0,0)$. There exist $\varepsilon>0$ and a constant $c>0$ such that for all $(x, \lambda) \in \mathbb{B}_{\varepsilon}((\bar{x}, \bar{\lambda}))$,

$$
\|x-\bar{x}\|+\operatorname{dist}(\lambda, \mathcal{M}(\bar{x}, 0,0)) \leq c\left\|\left(\begin{array}{c}
\nabla_{x} L(x, \lambda) \\
g(x)-\Pi_{\mathcal{K}}(\lambda+g(x))
\end{array}\right)\right\| .
$$

From $[20,22,7]$ we know that this local error bound plays a crucial role in analyzing the fast convergence rate of the SSQP and the augmented Lagrangian method.

Theorem 3.1 Let $(\bar{x}, \bar{\lambda})$ be a KKT point of $(1)$ with $(a, b)=(0,0)$. Property 1 holds at $(\bar{x}, \bar{\lambda})$ if and only if $\mathcal{S}_{\mathrm{KKT}}$ has the strong calmness at the origin for $(\bar{x}, \bar{\lambda})$.

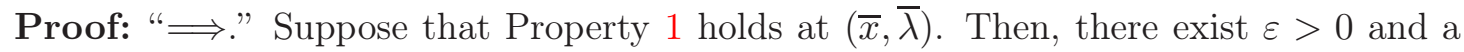
constant $c>0$ such that for all $(x, \lambda) \in \mathbb{B}_{\varepsilon}((\bar{x}, \bar{\lambda}))$,

$$
\|x-\bar{x}\|+\operatorname{dist}(\lambda, \mathcal{M}(\bar{x}, 0,0)) \leq c\left\|\left(\begin{array}{c}
\nabla_{x} L(x, \lambda) \\
g(x)-\Pi_{\mathcal{K}}(\lambda+g(x))
\end{array}\right)\right\| .
$$

Fix an arbitrary $\delta>0$. Take an arbitrary $(a, b) \in \mathbb{X} \times \mathbb{Y}$ with $\|(a, b)\| \leq \delta$. Let $\left(x^{\prime}, \lambda^{\prime}\right)$ be an arbitrary point from $\mathcal{S}_{\mathrm{KKT}}(a, b) \cap \mathbb{B}_{\varepsilon}((\bar{x}, \bar{\lambda}))$. From the last inequality, we have

$$
\left\|x^{\prime}-\bar{x}\right\|+\operatorname{dist}\left(\lambda^{\prime}, \mathcal{M}(\bar{x}, 0,0)\right) \leq c\left\|\left(\begin{array}{c}
\nabla_{x} L\left(x^{\prime}, \lambda^{\prime}\right) \\
g\left(x^{\prime}\right)-\Pi_{\mathcal{K}}\left(\lambda^{\prime}+g\left(x^{\prime}\right)\right)
\end{array}\right)\right\| .
$$

Since $\left(x^{\prime}, \lambda^{\prime}\right) \in \mathcal{S}_{\mathrm{KKT}}(a, b)$, we have $\nabla_{x} L\left(x^{\prime}, \lambda^{\prime}\right)=a$ and $g\left(x^{\prime}\right)-\Pi_{\mathcal{K}}\left(g\left(x^{\prime}\right)+\lambda^{\prime}-b\right)=b$. Together with the inequality (17) and the global Lipschitz continuity of $\Pi_{\mathcal{K}}$,

$$
\begin{aligned}
\left\|x^{\prime}-\bar{x}\right\|+\operatorname{dist}\left(\lambda^{\prime}, \mathcal{M}(\bar{x}, 0,0)\right) & \leq c\left\|\left(\begin{array}{c}
a \\
b+\Pi_{\mathcal{K}}\left(\lambda^{\prime}+g\left(x^{\prime}\right)-b\right)-\Pi_{\mathcal{K}}\left(\lambda^{\prime}+g\left(x^{\prime}\right)\right)
\end{array}\right)\right\| \\
& \leq 2 c\|(a, b)\| .
\end{aligned}
$$

From the arbitrariness of $\left(x^{\prime}, \lambda^{\prime}\right)$ in $\mathcal{S}_{\mathrm{KKT}}(a, b) \cap \mathbb{B}_{\varepsilon}((\bar{x}, \bar{\lambda}))$, we conclude that the multifunction $\mathcal{S}_{\mathrm{KKT}}$ has the strong calmness at the origin for $(\bar{x}, \bar{\lambda})$.

"£." Suppose that $\mathcal{S}_{\mathrm{KKT}}$ has the strong calmness at the origin for $(\bar{x}, \bar{\lambda})$. By Definition 1.1, there exist $\delta^{\prime}>0, \varepsilon^{\prime}>0$ and $\kappa^{\prime}>0$ such that for any $\left(a^{\prime}, b^{\prime}\right) \in \mathbb{X} \times \mathbb{Y}$ with $\left\|\left(a^{\prime}, b^{\prime}\right)\right\| \leq \delta^{\prime}$ and any $\left(x^{\prime}, \lambda^{\prime}\right) \in \mathcal{S}_{\mathrm{KKT}}\left(a^{\prime}, b^{\prime}\right) \cap \mathbb{B}_{\mathcal{\varepsilon}^{\prime}}((\bar{x}, \bar{\lambda}))$,

$$
\left\|x^{\prime}-\bar{x}\right\|+\operatorname{dist}\left(\lambda^{\prime}, \mathcal{M}(\bar{x}, 0,0)\right) \leq \kappa^{\prime}\left\|\left(a^{\prime}, b^{\prime}\right)\right\| .
$$


Notice that the functions $\nabla_{x} L(x, \lambda)$ and $g(x)-\Pi_{\mathcal{K}}(g(x)+\lambda)$ are continuous with respect to $(x, \lambda)$. There exist $\varepsilon>0$ and a constant $\gamma>0$ such that for any $(x, \lambda) \in \mathbb{B}_{\varepsilon}((\bar{x}, \bar{\lambda}))$,

$$
\begin{gathered}
\left\|\nabla_{x} L(x, \lambda)\right\|=\left\|\nabla_{x} L(x, \lambda)-\nabla_{x} L(\bar{x}, \bar{\lambda})\right\| \leq \frac{\delta^{\prime}}{2 \sqrt{2}}, \\
\left\|g(x)-\Pi_{\mathcal{K}}(g(x)+\lambda)\right\|=\left\|g(x)-\Pi_{\mathcal{K}}(g(x)+\lambda)-g(\bar{x})+\Pi_{\mathcal{K}}(g(\bar{x})+\bar{\lambda})\right\| \leq \min \left(\frac{\delta^{\prime}}{\sqrt{2}}, \frac{\varepsilon^{\prime}}{2}\right), \\
\|\nabla g(x)\| \leq \gamma \text { and }\left\|\nabla g(x)\left[g(x)-\Pi_{\mathcal{K}}(g(x)+\lambda)\right]\right\| \leq \frac{\delta^{\prime}}{2 \sqrt{2}} .
\end{gathered}
$$

Set $\epsilon=\min \left(\varepsilon^{\prime} / 2, \varepsilon\right)$. Fix an arbitrary point pair $(x, \lambda) \in \mathbb{B}_{\epsilon}((\bar{x}, \bar{\lambda}))$. Write $a=\nabla_{x} L(x, \lambda)$ and $b=g(x)-\Pi_{\mathcal{K}}(g(x)+\lambda)$. One may check that $(x, \lambda+b) \in S_{\mathrm{KKT}}(a+\nabla g(x) b, b)$. From $(x, \lambda) \in \mathbb{B}_{\epsilon}((\bar{x}, \bar{\lambda}))$ and the last inequalities, it follows that $\|(a+\nabla g(x) b, b)\| \leq \delta^{\prime}$. Also, from $(x, \lambda) \in \mathbb{B}_{\epsilon}((\bar{x}, \bar{\lambda}))$ and $\|b\| \leq \varepsilon^{\prime} / 2$, we have $\|(x, \lambda+b)-(\bar{x}, \bar{\lambda})\| \leq \varepsilon^{\prime}$. Now from $(x, \lambda+b) \in \mathbb{B}_{\varepsilon^{\prime}}((\bar{x}, \bar{\lambda})) \cap S_{\mathrm{KKT}}(a+\nabla g(x) b, b)$ and inequality (18), it follows that

$$
\|x-\bar{x}\|+\operatorname{dist}(\lambda+b, \mathcal{M}(\bar{x}, 0,0)) \leq \kappa^{\prime}\left\|\left(\begin{array}{c}
a+\nabla g(x) b \\
b
\end{array}\right)\right\|
$$

which, together with $a=\nabla_{x} L(x, \lambda)$ and $b=g(x)-\Pi_{\mathcal{K}}(g(x)+\lambda)$, implies that

$$
\begin{aligned}
\|x-\bar{x}\|+\operatorname{dist}(\lambda, \mathcal{M}(\bar{x}, 0,0)) & \leq\|x-\bar{x}\|+\operatorname{dist}(\lambda+b, \mathcal{M}(\bar{x}, 0,0))+\|b\| \\
& \leq \kappa^{\prime} \sqrt{2\left(\|\nabla g(x)\|^{2}+1\right)}\|(a, b)\|+\|b\| \\
& \leq\left(1+\kappa^{\prime} \sqrt{2 \gamma^{2}+2}\right)\left\|\left(\begin{array}{c}
\nabla_{x} L(x, \lambda) \\
g(x)-\Pi_{\mathcal{K}}(g(x)+\lambda)
\end{array}\right)\right\| .
\end{aligned}
$$

This, by the arbitrariness of $(x, \lambda)$ in $\mathbb{B}_{\epsilon}((\bar{x}, \bar{\lambda}))$, shows that Property 1 holds.

Remark 3.1 When $\mathcal{K}$ is specified as the nonpositive orthant cone in $\mathbb{R}^{m}$, the conclusion of Theorem 3.1 was obtained in [20, Remark 1] and [21, Remark 4] by invoking [12, Theorem 2]. In fact, from the proof of Theorem 3.1, it is not difficult to obtain the following conclusion: the multifunction $\mathcal{S}_{\mathrm{KKT}}$ is calm at the origin for $(\bar{x}, \bar{\lambda})$ if and only if there exist $\varepsilon>0$ and a constant $c>0$ such that for all $(x, \lambda) \in \mathbb{B}_{\varepsilon}((\bar{x}, \bar{\lambda}))$,

$$
\operatorname{dist}\left((x, \lambda), \mathcal{S}_{\mathrm{KKT}}(0,0)\right) \leq c\left\|\left(\begin{array}{c}
\nabla_{x} L(x, \lambda) \\
g(x)-\Pi_{\mathcal{K}}(\lambda+g(x))
\end{array}\right)\right\| .
$$

This partly extends the result of [12, Theorem 2] to nonpolyhedral conic optimization.

In order to provide the other equivalent characterization of the strong calmness, we need to introduce the concept of pseudo-isolated calmness of $\mathcal{X}_{\mathrm{KKT}}$.

Definition 3.1 Let $\bar{x}$ be a stationary point of $(1)$ with $(a, b)=(0,0)$ and $\bar{\lambda} \in \mathcal{M}(\bar{x}, 0,0)$. The multifunction $\mathcal{X}_{\mathrm{KKT}}$ is said to have the pseudo-isolated calmness at the origin for $\bar{x}$ if there exist $\varepsilon>0, \delta>0$ and a constant $\kappa>0$ such that for any $(a, b) \in \mathbb{X} \times \mathbb{Y}$ with $\|(a, b)\| \leq \delta$ and any $(x, \lambda) \in \mathcal{S}_{\mathrm{KKT}}(a, b) \cap \mathbb{B}_{\varepsilon}((\bar{x}, \bar{\lambda}))$, the following estimate holds

$$
\|x-\bar{x}\| \leq \kappa\|(a, b)\| .
$$


The reason why we call this property the pseudo-isolated calmness of $\mathcal{X}_{\mathrm{KKT}}$ is that it implies the isolated calmness of the multifunction $\mathcal{X}$ at $(\bar{\lambda}, 0,0)$ for $\bar{x}$ by the following proposition, but may not imply the isolatedness of $\bar{x}$ unless $\mathcal{M}(\bar{x}, 0,0)=\{\bar{\lambda}\}$. In fact, when $\mathcal{M}(\bar{x}, 0,0)=\{\bar{\lambda}\}$, under Robinson's CQ, the pseudo-isolated calmness of $\mathcal{X}_{\mathrm{KKT}}$ at the origin for $\bar{x}$ is equivalent to its isolated calmness at the origin for $\bar{x}$; see Appendix.

Proposition 3.1 Let $\bar{x}$ be a stationary point of $(1)$ with $(a, b)=(0,0)$ and $\bar{\lambda} \in \mathcal{M}(\bar{x}, 0,0)$.

(a) The multifunction $\mathcal{X}$ has the isolated calmness at $(\bar{\lambda}, 0,0)$ for $\bar{x}$ if and only if there exist $\varepsilon>0, \delta>0$ and $\kappa>0$ such that for any $(a, b) \in \mathbb{X} \times \mathbb{Y}$ with $\|(a, b)\| \leq \delta$ and any $(x, \lambda) \in \mathcal{S}_{\mathrm{KKT}}(a, b) \cap \mathbb{B}_{\varepsilon}((\bar{x}, \bar{\lambda}))$, the following estimate holds

$$
\|x-\bar{x}\| \leq \kappa\|(\lambda-\bar{\lambda}, a, b)\| .
$$

(b) The multifunction $\mathcal{X}$ has the isolated calmness at $(\bar{\lambda}, 0,0)$ for $\bar{x}$ if and only if $\xi=0$ is the unique solution to the following system

$$
\left\{\begin{array}{l}
\nabla_{x x}^{2} L(\bar{x}, \bar{\lambda}) \xi=0 \\
g^{\prime}(\bar{x}) \xi-\Pi_{\mathcal{K}}^{\prime}\left(g(\bar{x})+\bar{\lambda} ; g^{\prime}(\bar{x}) \xi\right)=0 .
\end{array}\right.
$$

Proof: $(\mathbf{a})^{\prime \prime} \Longrightarrow$ ". Suppose that the multifunction $\mathcal{X}$ has the isolated calmness at $(\bar{\lambda}, 0,0)$ for $\bar{x}$. Then, there exist $\varepsilon^{\prime}>0, \delta^{\prime}>0$ and $\kappa^{\prime}>0$ such that for any $(\lambda, a, b) \in \mathbb{B}_{\delta^{\prime}}((\bar{\lambda}, 0,0))$,

$$
\mathcal{X}(\lambda, a, b) \cap \mathbb{B}_{\mathcal{\varepsilon}^{\prime}}(\bar{x}) \subseteq\{\bar{x}\}+\kappa^{\prime}\|(\lambda-\bar{\lambda}, a, b)\| \mathbb{B}_{\mathbb{Y} \times \mathbb{X} \times \mathbb{Y}} .
$$

Set $\delta=\delta^{\prime} / 2$ and $\varepsilon=\min \left(\varepsilon^{\prime}, \delta^{\prime} / 2\right)$. Fix an arbitrary $(a, b) \in \mathbb{X} \times \mathbb{Y}$ with $\|(a, b)\| \leq \delta$. Pick up an arbitrary point $(x, \lambda) \in \mathcal{S}_{\mathrm{KKT}}(a, b) \cap \mathbb{B}_{\varepsilon}((\bar{x}, \bar{\lambda}))$. Clearly, $(\lambda, a, b) \in \mathbb{B}_{\delta^{\prime}}((\bar{\lambda}, 0,0))$ and $x \in \mathcal{X}(\lambda, a, b) \cap \mathbb{B}_{\varepsilon^{\prime}}(\bar{x})$. From the last inclusion, it immediately follows that

$$
\|x-\bar{x}\| \leq \kappa^{\prime}\|(\lambda-\bar{\lambda}, a, b)\| .
$$

"£". Suppose that there exist $\varepsilon>0, \delta>0$ and $\kappa>0$ such that for any $(a, b) \in \mathbb{X} \times \mathbb{Y}$ with $\|(a, b)\| \leq \delta$ and any $(x, \lambda) \in \mathcal{S}_{\mathrm{KKT}}(a, b) \cap \mathbb{B}_{\varepsilon}((\bar{x}, \bar{\lambda}))$, the estimate in (19) holds. Set $\delta^{\prime}=\min (\varepsilon / 2, \delta)$ and $\varepsilon^{\prime}=\varepsilon / 2$. Fix an arbitrary $(\lambda, a, b) \in \mathbb{B}_{\delta^{\prime}}((\bar{\lambda}, 0,0))$. Pick up an arbitrary $x \in \mathcal{X}(\lambda, a, b) \cap \mathbb{B}_{\varepsilon^{\prime}}(\bar{x})$. Clearly, $(x, \lambda) \in \mathcal{S}_{\mathrm{KKT}}(a, b) \cap \mathbb{B}_{\varepsilon}((\bar{x}, \bar{\lambda}))$. From (19), $\|x-\bar{x}\| \leq \kappa\|(\lambda-\bar{\lambda}, a, b)\|$, and hence $\mathcal{X}$ has the isolated calmness at $(\bar{\lambda}, 0,0)$ for $\bar{x}$.

(b) From the definition of $\mathcal{X}$ and $\mathcal{S}_{\mathrm{KKT}}$, it is immediate to obtain $(\lambda, a, b, x) \in \operatorname{gph} \mathcal{X}$ iff $\mathcal{A}(\lambda, a, b, x) \in \operatorname{gph} \mathcal{S}_{\mathrm{KKT}}$, where $\mathcal{A}: \mathbb{Y} \times \mathbb{X} \times \mathbb{Y} \times \mathbb{X} \rightarrow \mathbb{X} \times \mathbb{Y} \times \mathbb{X} \times \mathbb{Y}$ is defined by

$$
\mathcal{A}(\lambda, a, b, x)=(a, b, x, \lambda) .
$$

Clearly, the linear map $\mathcal{A}$ is invertible and gph $\mathcal{X}=\mathcal{A}^{-1}$ gph $\mathcal{S}_{\mathrm{KKT}}$. By [32, Exercise 6.7],

$$
\mathcal{T}_{\text {gph } \mathcal{X}}(\bar{\lambda}, 0,0, \bar{x})=\mathcal{A}^{-1} \mathcal{T}_{\text {gph } \mathcal{S}_{\text {KKT }}}(0,0, \bar{x}, \bar{\lambda}) .
$$


Hence, $\Delta x \in D \mathcal{X}((\bar{\lambda}, 0,0) \mid \bar{x})(\Delta \lambda, \Delta a, \Delta b)$ iff $(\Delta x, \Delta \lambda) \in D \mathcal{S}_{\mathrm{KKT}}((0,0) \mid(\bar{x}, \bar{\lambda}))(\Delta a, \Delta b)$. Together with the characterization of $D \mathcal{S}_{\mathrm{KKT}}((0,0) \mid(\bar{x}, \bar{\lambda}))$ in [9, Lemma 18 \& 19], it then follows that $\Delta x \in D \mathcal{X}((\bar{\lambda}, 0,0) \mid \bar{x})(\Delta \lambda, \Delta a, \Delta b)$ if and only if $(\Delta x, \Delta \lambda)$ satisfies

$$
\left\{\begin{array}{l}
\nabla_{x x}^{2} L(\bar{x}, \bar{\lambda}) \Delta x+g^{\prime}(\bar{x}) \Delta \lambda=0 \\
g^{\prime}(\bar{x}) \Delta x-\Pi_{\mathcal{K}}^{\prime}\left(g(\bar{x})+\bar{\lambda} ; g^{\prime}(\bar{x}) \Delta x+\Delta \lambda\right)=0
\end{array}\right.
$$

By Lemma 2.1, we conclude that $\mathcal{X}$ is isolated calm at $(\bar{\lambda}, 0,0)$ for $\bar{x}$ if and only if system (20) has the unique solution $\xi=0$. The proof is then completed.

It is worthwhile to mention that for the case that $\mathcal{K}$ is a semidefinite positive cone, Zhang and Zhang [35] proved that the estimate in (19) holds iff (20) has only the trivial solution $\xi=0$. Here, we associate this property with the isolated calmness of $\mathcal{X}$.

In addition, we also need the following lemma which states that the calmness of $\mathcal{M}$ at $(\bar{x}, 0,0)$ for $\bar{\lambda} \in \mathcal{M}(\bar{x}, 0,0)$ is equivalent to that of $\mathcal{G}_{\bar{x}}$ at $(\nabla f(\bar{x}), g(\bar{x}))$ for $\bar{\lambda}$.

Lemma 3.1 Let $(\bar{x}, \bar{\lambda})$ be a KKT point of the problem $(1)$ with $(a, b)=(0,0)$. Then, $\mathcal{M}$ is calm at $(\bar{x}, 0,0)$ for $\bar{\lambda}$ if and only if $\mathcal{G}_{\bar{x}}$ is calm at $(\nabla f(\bar{x}), g(\bar{x}))$ for $\bar{\lambda}$.

Proof: Suppose that the multiplier set mapping $\mathcal{M}$ is calm at $(\bar{x}, 0,0)$ for $\bar{\lambda}$. Then, there exist $\varepsilon_{1}>0, \varepsilon_{2}>0$ and $\kappa_{1}>0$ such that for all $(x, a, b) \in \mathbb{B}_{\varepsilon_{1}}((\bar{x}, 0,0))$,

$$
\mathcal{M}(x, a, b) \cap \mathbb{B}_{\varepsilon_{2}}(\bar{\lambda}) \subseteq \mathcal{M}(\bar{x}, 0,0)+\kappa_{1}\|(x, a, b)-(\bar{x}, 0,0)\| \mathbb{B}_{\mathbb{X} \times \mathbb{X} \times \mathbb{Y}} .
$$

Write $\bar{\eta}=\nabla f(\bar{x})$ and $\bar{y}=g(\bar{x})$. Fix an arbitrary $(\eta, y) \in \mathbb{B}_{\varepsilon_{1}}((\bar{\eta}, \bar{y}))$. Pick up an arbitrary $\lambda \in \mathcal{G}_{\bar{x}}(\eta, y) \cap \mathbb{B}_{\varepsilon_{2}}(\bar{\lambda})$. It is easy to check that $\lambda \in \mathcal{M}(\bar{x}, a, b)$ with $a=\bar{\eta}-\eta$ and $b=\bar{y}-y$. Clearly, $(\bar{x}, a, b) \in \mathbb{B}_{\varepsilon_{1}}((\bar{x}, 0,0))$. From the last equation, it follows that

$$
\operatorname{dist}\left(\lambda, \mathcal{G}_{\bar{x}}(\bar{\eta}, \bar{y})\right)=\operatorname{dist}(\lambda, \mathcal{M}(\bar{x}, 0,0)) \leq \kappa\|(a, b)-(0,0)\|=\|(\eta, y)-(\bar{\eta}, \bar{y})\| .
$$

By the arbitrariness of $\lambda$ in $\mathcal{G}_{\bar{x}}(\eta, y) \cap \mathbb{B}_{\varepsilon_{2}}(\bar{\lambda})$, this shows that $\mathcal{G}_{\bar{x}}$ is calm at $(\bar{\eta}, \bar{y})$ for $\bar{\lambda}$.

Conversely, suppose that the multifunction $\mathcal{G}_{\bar{x}}$ is calm at $(\bar{\eta}, \bar{y})$ for $\bar{\lambda}$. Then there exist $\delta_{1}>0, \delta_{2}>0$ and a constant $\nu>0$ such that for any $(\eta, y) \in \mathbb{B}_{\delta_{1}}((\bar{\eta}, \bar{y}))$,

$$
\mathcal{G}_{\bar{x}}(\eta, y) \cap \mathbb{B}_{\delta_{2}}(\bar{\lambda}) \subseteq \mathcal{G}_{\bar{x}}\left((\bar{\eta}, \bar{y})+\nu\|(\eta, y)-(\bar{\eta}, \bar{y})\| \mathbb{B}_{\mathbb{X} \times \mathbb{Y}}\right.
$$

Since the mappings $\nabla f, g$ and $\nabla g$ are locally Lipschitz continuous at $\bar{x}$, there exist $\widehat{\delta}_{1}>0$, $\widehat{\delta}_{2}>0$ and a constant $\widehat{\nu}>0$ such that for all $x \in \mathbb{B}_{\widehat{\delta}_{1}}(\bar{x})$ and $\lambda \in \mathbb{B}_{\widehat{\delta}_{2}}(\bar{\lambda})$,

$$
\|\nabla f(x)-\nabla f(\bar{x})\|+\|g(x)-g(\bar{x})\|+\|[\nabla g(x)-\nabla g(\bar{x})] \lambda\| \leq \widehat{\nu}\|x-\bar{x}\| .
$$

Set $\varepsilon_{1}=\min \left(\frac{\delta_{1}}{\hat{\nu}+1}, \widehat{\delta}_{1}\right)$ and $\varepsilon_{2}=\min \left(\widehat{\delta}_{2}, \delta_{2}\right)$. Fix an arbitrary $(x, a, b) \in \mathbb{B}_{\varepsilon_{1}}((\bar{x}, 0,0))$. Take an arbitrary point $\lambda \in \mathcal{M}(x, a, b) \cap \mathbb{B}_{\varepsilon_{2}}(\bar{\lambda})$. Then $\lambda \in \mathcal{G}_{\bar{x}}(\eta, y)$ with $y=g(x)-b$ and $\eta=\nabla f(x)-a+[\nabla g(x)-\nabla g(\bar{x})] \lambda$. Also, by using the inequality (22) one may obtain that $\|(\eta, y)-(\bar{\eta}, \bar{y})\| \leq \widehat{\nu}\|x-\bar{x}\|+\|(a, b)\| \leq \delta_{1}$. Thus, from (21) it follows that

$$
\begin{aligned}
\operatorname{dist}\left(\lambda, \mathcal{G}_{\bar{x}}(\bar{\eta}, \bar{y})\right) & \leq \nu\|(\eta, y)-(\bar{\eta}, \bar{y})\| \leq \nu(\widehat{\nu}\|x-\bar{x}\|+\|(a, b)\|) \\
& \leq \sqrt{2} \nu \max (\widehat{\nu}, 1)\|(x, a, b)-(\bar{x}, 0,0)\| .
\end{aligned}
$$

By the arbitrariness of $\lambda$ in $\mathcal{M}(x, a, b) \cap \mathbb{B}_{\varepsilon_{2}}(\bar{\lambda}), \mathcal{M}$ is calm at $(\bar{x}, 0,0)$ for $\bar{\lambda}$. 
Theorem 3.2 Let $(\bar{x}, \bar{\lambda})$ be a KKT point of $(1)$ with $(a, b)=(0,0)$ and $\mathcal{M}(\bar{x}, 0,0) \neq\{\bar{\lambda}\}$. Then, $\mathcal{S}_{\mathrm{KKT}}$ has the strong calmness at the origin for $(\bar{x}, \bar{\lambda})$ if and only if $\mathcal{X}_{\mathrm{KKT}}$ has the pseudo-isolated calmness at the origin for $\bar{x}$ and $\mathcal{M}$ has the calmness at $(\bar{x}, 0,0)$ for $\bar{\lambda}$.

Proof: "£". Write $\bar{\eta}=\nabla f(\bar{x})$ and $\bar{y}=g(\bar{x})$. By Lemma 3.1, $\mathcal{G}_{\bar{x}}$ is calm at $(\bar{\eta}, \bar{y})$ for $\bar{\lambda}$. Then, there exist $\delta^{\prime}>0, \varepsilon^{\prime}>0$ and $\kappa^{\prime}>0$ such that for all $(\eta, y) \in \mathbb{B}_{\varepsilon^{\prime}}((\bar{\eta}, \bar{y}))$,

$$
\mathcal{G}_{\bar{x}}(\eta, y) \cap \mathbb{B}_{\delta^{\prime}}(\bar{\lambda}) \subseteq \mathcal{G}_{\bar{x}}(\bar{\eta}, \bar{y})+\kappa^{\prime}\|(\eta, y)-(\bar{\eta}, \bar{y})\| \mathbb{B}_{\mathbb{X} \times \mathbb{Y}}
$$

Since $\mathcal{X}_{\mathrm{KKT}}$ has the pseudo-isolated calmness at the origin for $\bar{x}$, there exist $\widetilde{\delta}>0, \widetilde{\varepsilon}>0$ and $\widetilde{\kappa}>0$ such that for any $(\widetilde{a}, \widetilde{b}) \in \mathbb{X} \times \mathbb{Y}$ with $\|(\widetilde{a}, \widetilde{b})\| \leq \widetilde{\delta}$ and any $(\widetilde{x}, \widetilde{\lambda}) \in \mathbb{B}_{\widetilde{\varepsilon}}((\bar{x}, \bar{\lambda}))$,

$$
\|x-\bar{x}\| \leq \widetilde{\kappa}\|(\widetilde{a}, \widetilde{b})\|
$$

From the local Lipschitz continuity of $\nabla f(\cdot)$ and $g(\cdot)$, there exist $\widehat{\varepsilon}>0$ and constants $c_{1}>0$ and $c_{2}>0$ such that for all $(x, \lambda) \in \mathbb{B}_{\widehat{\varepsilon}}((\bar{x}, \bar{\lambda}))$, the following inequalities hold:

$$
\left\{\begin{array}{l}
\|\nabla f(x)+\nabla g(x) \lambda-\nabla g(\bar{x}) \lambda-\nabla f(\bar{x})\| \leq c_{1}\|x-\bar{x}\|, \\
\|g(x)-g(\bar{x})\| \leq c_{2}\|x-\bar{x}\|,\|g(x)-g(\bar{x})+b\| \leq \frac{\varepsilon^{\prime}}{\sqrt{2}},\|\lambda-\bar{\lambda}\| \leq \delta^{\prime}, \\
\|\nabla f(x)+\nabla g(x) \lambda-\nabla g(\bar{x}) \lambda-\nabla f(\bar{x})-a\| \leq \frac{\varepsilon^{\prime}}{\sqrt{2}} .
\end{array}\right.
$$

Set $\delta=\min \left(\varepsilon^{\prime} / 2, \widetilde{\delta}\right)$ and $\varepsilon=\min (\widetilde{\varepsilon}, \widehat{\varepsilon})$. Fix an arbitrary $(a, b) \in \mathbb{X} \times \mathbb{Y}$ with $\|(a, b)\| \leq \delta$. Pick up an arbitrary $(x, \lambda) \in \mathcal{S}_{\mathrm{KKT}}(a, b) \cap \mathbb{B}_{\varepsilon}((\bar{x}, \bar{\lambda}))$. We check that $\lambda \in \mathcal{G}_{\bar{x}}\left(\eta^{\prime}, y^{\prime}\right)$ with $\eta^{\prime}=\nabla f(x)+\nabla g(x) \lambda-a-\nabla g(\bar{x}) \lambda$ and $y^{\prime}=g(x)-b$. Clearly, $\lambda \in \mathbb{B}_{\delta^{\prime}}(\bar{\lambda})$. Also, from the inequalities in (25b)-(25c), we have $\left(\eta^{\prime}, y^{\prime}\right) \in \mathbb{B}_{\varepsilon^{\prime}}((\bar{\eta}, \bar{y}))$. By (23) it follows that

$$
\begin{aligned}
\operatorname{dist}(\lambda, \mathcal{M}(\bar{x}, 0,0)) & =\operatorname{dist}\left(\lambda, \mathcal{G}_{\bar{x}}(\bar{\eta}, \bar{y})\right) \leq \kappa^{\prime}\left\|\left(\eta^{\prime}, y^{\prime}\right)-(\nabla f(\bar{x}), g(\bar{x}))\right\| \\
& =\kappa^{\prime}\left\|\left(\begin{array}{c}
\nabla f(x)+\nabla g(x) \lambda-a-\nabla g(\bar{x}) \lambda-\nabla f(\bar{x}) \\
g(x)-b-g(\bar{x})
\end{array}\right)\right\| \\
& \leq \kappa^{\prime}\left[\sqrt{2 c_{1}^{2}+2 c_{2}^{2}}\|x-\bar{x}\|+\|(a, b)\|\right] \\
& \leq \kappa^{\prime}\left[\widetilde{\kappa} \sqrt{2 c_{1}^{2}+2 c_{2}^{2}}\|(a, b)\|+\|(a, b)\|\right]
\end{aligned}
$$

where the last equality is due to (24) implied by $\|(a, b)\| \leq \delta \leq \widetilde{\delta}$ and $(x, \lambda) \in \mathbb{B}_{\widetilde{\varepsilon}}((\bar{x}, \bar{\lambda}))$. This shows that $\mathcal{S}_{\mathrm{KKT}}$ has the strong calmness at the origin for $(\bar{x}, \bar{\lambda})$.

“”". Suppose that $\mathcal{S}_{\mathrm{KKT}}$ has the strong calmness at the origin for $(\bar{x}, \bar{\lambda})$. Clearly, $\mathcal{X}_{\text {KKT }}$ has the pseudo-isolated calmness at the origin for $\bar{x}$. It suffices to prove that $\mathcal{M}$ is calm at $(\bar{x}, 0,0)$ for $\bar{\lambda}$. By the strong calmness of $\mathcal{S}_{\mathrm{KKT}}$ at the origin for $(\bar{x}, \bar{\lambda})$, there exist $\varepsilon>0, \delta>0$ and $\kappa>0$ such that for any $(a, b) \in \mathbb{X} \times \mathbb{Y}$ with $\|(a, b)\| \leq \delta$ and any $(x, \lambda) \in \mathcal{S}_{\mathrm{KKT}}(a, b) \cap \mathbb{B}_{\varepsilon}((\bar{x}, \bar{\lambda}))$, the following estimate holds:

$$
\|x-\bar{x}\|+\operatorname{dist}(\lambda, \mathcal{M}(\bar{x}, 0,0)) \leq \kappa\|(a, b)\| .
$$


Set $\varepsilon^{\prime}=\frac{1}{\sqrt{2}} \min (\varepsilon, \delta)$ and $\delta^{\prime}=\frac{1}{\sqrt{2}} \varepsilon$. Fix an arbitrary $(x, a, b) \in \mathbb{B}_{\varepsilon^{\prime}}((\bar{x}, 0,0))$. Pick up an arbitrary $\lambda \in \mathcal{M}(x, a, b) \cap \mathbb{B}_{\delta^{\prime}}(\bar{\lambda})$. Clearly, $(x, \lambda) \in \mathcal{S}_{\mathrm{KKT}}(a, b) \cap \mathbb{B}_{\varepsilon}((\bar{x}, \bar{\lambda}))$. By $(26)$,

$$
\operatorname{dist}(\lambda, \mathcal{M}(\bar{x}, 0,0)) \leq \kappa\|(a, b)\| \leq \kappa\|(x-\bar{x}, a, b)\| .
$$

This shows that $\mathcal{M}$ is calm at $(\bar{x}, 0,0)$ for $\bar{\lambda}$. Thus, we complete the proof.

\section{Pseudo-isolated calmness of $\mathcal{X}_{\mathrm{KKT}}$}

We shall focus on the characterizations of the pseudo-isolated calmness of $\mathcal{X}_{\mathrm{KKT}}$ in terms of the noncriticality of the associated multiplier. Along with Theorem 3.2, some sufficient characterizations are also obtained for the strong calmness of $\mathcal{S}_{\mathrm{KKT}}$.

Proposition 4.1 Let $(\bar{x}, \bar{\lambda})$ be a KKT point of the problem (1) with $(a, b)=(0,0)$. If $\mathcal{X}_{\mathrm{KKT}}$ has the pseudo-isolated calmness at the origin for $\bar{x}$, then $\bar{\lambda}$ is noncritical.

Proof: Let $\widetilde{\mathcal{S}}_{\mathrm{KKT}}(a, b):=\left\{(x, \lambda) \mid \nabla f(x)+\nabla g(x)(\lambda+b)=a, g(x)-\Pi_{\mathcal{K}}(g(x)+\lambda)=b\right\}$ for $(a, b) \in \mathbb{X} \times \mathbb{Y}$. One may check that $(x, \lambda) \in \mathcal{S}_{\mathrm{KKT}}(a, b)$ iff $(x, \lambda-b) \in \widetilde{\mathcal{S}}_{\mathrm{KKT}}(a, b)$. By the given assumption and Definition 3.1, there exist $\varepsilon^{\prime}>0, \delta^{\prime}>0$ and $\kappa^{\prime}>0$ such that for any $(a, b) \in \mathbb{X} \times \mathbb{Y}$ with $\|(a, b)\| \leq \delta^{\prime}$ and any $(x, \lambda) \in \widetilde{\mathcal{S}}_{\mathrm{KKT}}(a, b) \cap \mathbb{B}_{\mathcal{\varepsilon}^{\prime}}((\bar{x}, \bar{\lambda}))$,

$$
\|x-\bar{x}\| \leq \kappa^{\prime}\|(a, b)\| .
$$

Let $\left(\xi^{*}, v^{*}\right) \in \mathbb{X} \times \mathbb{Y}$ be an arbitrary solution of system (10). For any sufficiently small $t>0$, define $x_{t}:=\bar{x}+t \xi^{*}$ and $\lambda_{t}:=\bar{\lambda}+t v^{*}$. By the directional differentiability of $\Pi_{\mathcal{K}}$,

$$
\begin{aligned}
\Pi_{\mathcal{K}}\left(g\left(x_{t}\right)+\lambda_{t}\right) & =\Pi_{\mathcal{K}}\left(g(\bar{x})+t g^{\prime}(\bar{x}) \xi^{*}+\bar{\lambda}+t v^{*}+o(t)\right) \\
& =\Pi_{\mathcal{K}}(g(\bar{x})+\bar{\lambda})+t \Pi_{\mathcal{K}}^{\prime}\left(g(\bar{x})+\bar{\lambda} ; g^{\prime}(\bar{x}) \xi^{*}+v^{*}\right)+o(t) \\
& =g(\bar{x})+t g^{\prime}(\bar{x}) \xi^{*}+o(t)
\end{aligned}
$$

where the last equality is due to the fact that $\left(\xi^{*}, v^{*}\right)$ is a solution of the system (10) and $g(\bar{x})=\Pi_{\mathcal{K}}(g(\bar{x})+\bar{\lambda})$. Together with $g\left(x_{t}\right)=g(\bar{x})+t g^{\prime}(\bar{x}) \xi^{*}+o(t)$, it follows that

$$
b_{t}:=g\left(x_{t}\right)-\Pi_{\mathcal{K}}\left(g\left(x_{t}\right)+\lambda_{t}\right)=o(t) .
$$

In addition, from $\nabla f(\bar{x})+\nabla g(\bar{x}) \bar{\lambda}=0$ and $\nabla^{2} f(\bar{x}) \xi^{*}+\nabla(\nabla g(\cdot) \bar{\lambda})(\bar{x}) \xi^{*}=\nabla_{x x}^{2} L(\bar{x}, \bar{\lambda}) \xi^{*}$,

$$
\begin{aligned}
a_{t} & :=\nabla f\left(x_{t}\right)+\nabla g\left(x_{t}\right)\left(\lambda_{t}+b_{t}\right) \\
& =\nabla f(\bar{x})+t \nabla^{2} f(\bar{x}) \xi^{*}+\left(\nabla g(\bar{x})+t \nabla(\nabla g(\cdot))(\bar{x}) \xi^{*}+o(t)\right)\left(\bar{\lambda}+t v^{*}+b_{t}\right)+o(t) \\
& =t \nabla_{x x}^{2} L(\bar{x}, \bar{\lambda}) \xi^{*}+t \nabla g(\bar{x}) v^{*}+o(t)=o(t)
\end{aligned}
$$

where the last equality is using the fact that $\left(\xi^{*}, v^{*}\right)$ is the solution of (10). The last two equations show that, for all sufficiently small $t>0,\left(x_{t}, \lambda_{t}\right) \in \widetilde{\mathcal{S}}_{\mathrm{KKT}}\left(a_{t}, b_{t}\right) \cap \mathbb{B}_{\varepsilon^{\prime}}(\bar{x}, \bar{\lambda})$ with $\left\|\left(a_{t}, b_{t}\right)\right\| \leq \delta^{\prime}$. From (27), for all sufficiently small $t>0$, it holds that

$$
\left\|\xi^{*}\right\| \leq \kappa^{\prime}\left\|\left(a_{t}, b_{t}\right)\right\| / t \rightarrow 0 .
$$


This implies $\xi^{*}=0$. By Proposition 2.1, $\bar{\lambda}$ is noncritical for $(1)$ with $(a, b)=(0,0)$.

Proposition 4.1 shows that the pseudo-isolated calmness of $\mathcal{X}_{\mathrm{KKT}}$ implies the noncriticality of the associated multiplier. However, its converse conclusion generally does not hold unless $\mathcal{K}$ is polyhedral. Motivated by [7, Theorem 3.2] and [35, Theorem 3.3], we establish the converse conclusion of Proposition 4.1 under an additional condition.

Proposition 4.2 Let $(\bar{x}, \bar{\lambda})$ be a KKT point of $(1)$ with $(a, b)=(0,0)$. Define the sets

$$
\begin{gathered}
\Sigma(\bar{x}, \bar{\lambda}):=\left\{(\xi, \zeta) \in \mathbb{X} \times \mathbb{Y}: \nabla_{x x}^{2} L(\bar{x}, \bar{\lambda}) \xi+\nabla g(\bar{x})\left[\zeta+\frac{1}{2} \nabla \Upsilon\left(g^{\prime}(\bar{x}) \xi\right)\right]=0\right\}, \\
\Gamma(\bar{x}, \bar{\lambda}):=\left\{(\xi, \zeta) \in \mathbb{X} \times \mathbb{Y}: g^{\prime}(\bar{x}) \xi \in \mathcal{C}_{\mathcal{K}}(g(\bar{x}), \bar{\lambda}), \zeta \in\left[\mathcal{C}_{\mathcal{K}}(g(\bar{x}), \bar{\lambda})\right]^{\circ}\right\} .
\end{gathered}
$$

Suppose that $\left\langle g^{\prime}(\bar{x}) \xi, \zeta\right\rangle \geq 0$ for any $(\xi, \zeta) \in \Sigma(\bar{x}, \bar{\lambda}) \cap \Gamma(\bar{x}, \bar{\lambda})$ and $\nabla g(\bar{x})\left[\mathcal{C}_{\mathcal{K}}(g(\bar{x}), \bar{\lambda})\right]^{\circ}$ is closed. Then, under the noncriticality of the Lagrange multiplier $\bar{\lambda}$ for the problem (1) with $(a, b)=(0,0), \mathcal{X}_{\mathrm{KKT}}$ has the pseudo-isolated calmness at the origin for $\bar{x}$.

Proof: Suppose on the contradiction that $\mathcal{X}_{\mathrm{KKT}}$ does not have the pseudo-isolated calmness at the origin for $\bar{x}$. By Definition 3.1, there exist sequences $\left\{\left(a^{k}, b^{k}\right)\right\} \rightarrow 0$ and $\left\{\left(x^{k}, \lambda^{k}\right)\right\} \rightarrow(\bar{x}, \bar{\lambda})$ with $\left(x^{k}, \lambda^{k}\right) \in \mathcal{S}_{\mathrm{KKT}}\left(a^{k}, b^{k}\right)$ for each $k \in \mathbb{N}$ such that $\frac{\left\|\left(a^{k}, b^{k}\right)\right\|}{\left\|x^{k}-\bar{x}\right\|} \rightarrow 0$ as $k \rightarrow \infty$. For convenience, we write $t_{k}:=\left\|x^{k}-\bar{x}\right\|$ for each $k$, and assume (if necessary taking a subsequence) that $\frac{x^{k}-\bar{x}}{t_{k}} \rightarrow \xi$ for some $\xi \in \mathbb{X}$ with $\|\xi\|=1$ when $k \rightarrow \infty$. From $\left(x^{k}, \lambda^{k}\right) \in \mathcal{S}_{\mathrm{KKT}}\left(a^{k}, b^{k}\right)$ and the definition of $\mathcal{S}_{\mathrm{KKT}}$, it immediately follows that

$$
\nabla f\left(x^{k}\right)+\nabla g\left(x^{k}\right) \lambda^{k}=a^{k} \text { and } \lambda^{k} \in \mathcal{N}_{\mathcal{K}}\left(g\left(x^{k}\right)-b^{k}\right) .
$$

By using the twice continuous differentiability of $f$ and $g$, for each $k \in \mathbb{N}$ we have

$$
\begin{aligned}
\nabla f\left(x^{k}\right) & =\nabla f(\bar{x})+\nabla^{2} f(\bar{x})\left(x^{k}-\bar{x}\right)+o\left(t_{k}\right), \\
g^{\prime}\left(x^{k}\right) & =g^{\prime}(\bar{x})+g^{\prime \prime}(\bar{x})\left(x^{k}-\bar{x}\right)+o\left(t_{k}\right) .
\end{aligned}
$$

Together with the first equality in $(29)$ and $\nabla f(\bar{x})+\nabla g(\bar{x}) \bar{\lambda}=0$, we immediately obtain

$$
a^{k}=\nabla^{2} f(\bar{x})\left(x^{k}-\bar{x}\right)+\nabla g(\bar{x})\left(\lambda^{k}-\bar{\lambda}\right)+\left[g^{\prime \prime}(\bar{x})\left(x^{k}-\bar{x}\right)\right]^{*} \lambda^{k}+o\left(t_{k}\right) .
$$

By dividing the two sides of (30) with $t_{k}$ and taking the limit $k \rightarrow \infty$, we have

$$
w:=\lim _{k \rightarrow \infty} \frac{\nabla g(\bar{x})\left(\lambda^{k}-\bar{\lambda}\right)}{t_{k}}=-\nabla_{x x}^{2} L(\bar{x}, \bar{\lambda}) \xi
$$

where the equality is using $\lim _{k \rightarrow \infty} a^{k} / t_{k}=0$ and $\lim _{k \rightarrow \infty} \lambda^{k}=\bar{\lambda}$. Write $\bar{y}:=g(\bar{x})$. Since $g\left(x^{k}\right)=\bar{y}+t_{k} g^{\prime}(\bar{x}) \xi+o\left(t_{k}\right)$ and $b^{k}=o\left(t_{k}\right)$, it then follows that

$$
g\left(x^{k}\right)-b^{k}=\bar{y}+t_{k} u^{k} \text { with } u^{k}:=g^{\prime}(\bar{x}) \xi+o\left(t_{k}\right) / t_{k} .
$$

Since $\lambda^{k} \in \mathcal{N}_{\mathcal{K}}\left(\bar{y}+t_{k} u^{k}\right)$ for each $k$, by the conic reducibility of $\mathcal{K}$ and Lemma 2.2, for each sufficiently large $k$ there exist $\mu^{k} \in \mathcal{N}_{D}\left(\Xi\left(\bar{y}+t_{k} u^{k}\right)\right)$ such that $\lambda^{k}=\nabla \Xi\left(\bar{y}+t_{k} u^{k}\right) \mu^{k}$. 
Notice that $\nabla \Xi(\bar{y}): \mathbb{Y} \rightarrow \mathbb{Z}$ is injective. From $\lambda^{k}=\nabla \Xi\left(\bar{y}+t_{k} u^{k}\right) \mu^{k}$ and the convergence of $\lambda^{k}$, the sequence $\left\{\mu^{k}\right\}$ is bounded. We may assume (taking a subsequence if necessary) that $\mu^{k} \rightarrow \bar{\mu}$. Taking the limit $k \rightarrow \infty$ to the equality $\lambda^{k}=\nabla \Xi\left(\bar{y}+t_{k} u^{k}\right) \mu^{k}$ yields that $\bar{\lambda}=\nabla \Xi(\bar{y}) \bar{\mu}$. Next, we proceed the arguments by three steps as shown below.

Step 1: $g^{\prime}(\bar{x}) \xi \in \mathcal{C}_{\mathcal{K}}(g(\bar{x}), \bar{\lambda})$. Since $g\left(x^{k}\right)-b^{k} \in \mathcal{K}$ and $g(\bar{x}) \in \mathcal{K}$, we immediately have

$$
\mathcal{T}_{\mathcal{K}}(g(\bar{x})) \ni g\left(x^{k}\right)-b^{k}-g(\bar{x})=g^{\prime}(\bar{x})\left(x^{k}-\bar{x}\right)+o\left(t_{k}\right),
$$

which implies $g^{\prime}(\bar{x}) \xi \in \mathcal{T}_{\mathcal{K}}(g(\bar{x}))$. Since $0=\left\langle\mu^{k}, \Xi\left(\bar{y}+t_{k} u^{k}\right)\right\rangle=\left\langle\mu^{k}, t_{k} \Xi^{\prime}(\bar{y}) u^{k}+o\left(t_{k}\right)\right\rangle$ for all sufficiently large $k$, dividing the two sides of this equality by $t_{k}$ and taking the limit $k \rightarrow \infty$ yields that $0=\left\langle\nabla \Xi(\bar{y}) \bar{\mu}, g^{\prime}(\bar{x}) \xi\right\rangle=\left\langle\bar{\lambda}, g^{\prime}(\bar{x}) \xi\right\rangle$. Together with $g^{\prime}(\bar{x}) \xi \in \mathcal{T}_{\mathcal{K}}(g(\bar{x}))$, we obtain that $g^{\prime}(\bar{x}) \xi \in \mathcal{C}_{\mathcal{K}}(g(\bar{x}), \bar{\lambda})$. The proof of this step is completed.

Step 2: $w=\nabla g(\bar{x}) v$ for some $v \in \mathbb{Y}$ with $v-\frac{1}{2} \nabla \Upsilon\left(g^{\prime}(\bar{x}) \xi\right) \in\left[\mathcal{C}_{\mathcal{K}}(g(\bar{x}), \bar{\lambda})\right]^{\circ}$. Note that

$$
\begin{aligned}
\nabla g(\bar{x}) \frac{\lambda^{k}-\bar{\lambda}}{t_{k}} & =\nabla g(\bar{x}) \frac{\left(\nabla \Xi\left(\bar{y}+t_{k} u^{k}\right)-\nabla \Xi(\bar{y})\right) \mu^{k}+\nabla \Xi(\bar{y})\left(\mu^{k}-\bar{\mu}\right)}{t_{k}} \\
& =\nabla g(\bar{x}) \nabla\left(\nabla \Xi(\cdot) \mu^{k}\right)(\bar{y}) u^{k}+\nabla g(\bar{x}) \nabla \Xi(\bar{y}) \frac{\mu^{k}-\bar{\mu}}{t_{k}} .
\end{aligned}
$$

By recalling $w=\lim _{k \rightarrow \infty} \frac{\nabla g(\bar{x})\left(\lambda^{k}-\bar{\lambda}\right)}{t_{k}}$ and taking the limit $k \rightarrow \infty$ to the both sides,

$$
\lim _{k \rightarrow \infty} \nabla g(\bar{x}) \nabla \Xi(\bar{y}) \frac{\mu^{k}-\bar{\mu}}{t_{k}}=w-\nabla g(\bar{x}) \nabla(\nabla \Xi(\cdot) \bar{\mu})(\bar{y})\left(g^{\prime}(\bar{x}) \xi\right) .
$$

We next argue that $\nabla \Xi(\bar{y}) \frac{\mu^{k}-\bar{\mu}}{t_{k}} \in\left[\mathcal{C}_{\mathcal{K}}(\bar{y}, \bar{\lambda})\right]^{\circ}$ for each $k \in \mathbb{N}$. Fix an arbitrary $k \in \mathbb{N}$. Take an arbitrary $d \in \mathcal{C}_{\mathcal{K}}(\bar{y}, \bar{\lambda})$. Then $\langle d, \bar{\lambda}\rangle=0$ by recalling that $\mathcal{C}_{\mathcal{K}}(\bar{y}, \bar{\lambda})=\mathcal{T}_{\mathcal{K}}(\bar{y}) \cap \llbracket \bar{\lambda} \rrbracket^{\perp}$. Also, by Lemma $2.2, \mathcal{K} \cap \mathcal{Y}=\Xi^{-1}(D)$. Together with the surjectivity of $\Xi^{\prime}(\bar{y}): \mathbb{Y} \rightarrow \mathbb{Z}$ and [32, Exercise 6.7], $d \in \mathcal{T}_{\mathcal{K}}(\bar{y})=\left[\Xi^{\prime}(\bar{y})\right]^{-1} \mathcal{T}_{D}(\Xi(\bar{y}))=\left[\Xi^{\prime}(\bar{y})\right]^{-1} D$, and consequently

$$
\left\langle d, \nabla \Xi(\bar{y})\left(\mu^{k}-\bar{\mu}\right)\right\rangle=\left\langle\Xi^{\prime}(\bar{y}) d, \mu^{k}\right\rangle-\langle d, \bar{\lambda}\rangle=\left\langle\Xi^{\prime}(\bar{y}) d, \mu^{k}\right\rangle \leq 0,
$$

where the inequality is using $\Xi^{\prime}(\bar{y}) d \in D$ and $\mu^{k} \in D^{\circ}$. So, the stated inclusion holds. From the given assumption, the set $\nabla g(\bar{x})\left[\mathcal{C}_{\mathcal{K}}(\bar{y}, \bar{\lambda})\right]^{\circ}$ is closed. Then, from (32) there exists $\eta \in\left[\mathcal{C}_{\mathcal{K}}(\bar{y}, \bar{\lambda})\right]^{\circ}$ such that $\lim _{k \rightarrow \infty} \nabla g(\bar{x}) \nabla \Xi(\bar{y}) \frac{\mu^{k}-\bar{\mu}}{t_{k}}=\nabla g(\bar{x}) \eta$. Along with (32),

$$
w=\nabla g(\bar{x}) v \quad \text { with } \quad v-\nabla(\nabla \Xi(\cdot) \bar{\mu})(\bar{y})\left(g^{\prime}(\bar{x}) \xi\right)=\eta \in\left[\mathcal{C}_{\mathcal{K}}(\bar{y}, \bar{\lambda})\right]^{\circ} .
$$

Recall that $\Upsilon(h)=\left\langle\bar{\mu}, \Xi^{\prime \prime}(\bar{y})(h, h)\right\rangle$ for $h \in \mathcal{C}_{\mathcal{K}}(\bar{y}, \bar{\lambda})$. From $g^{\prime}(\bar{x}) \xi \in \mathcal{C}_{\mathcal{K}}(\bar{y}, \bar{\lambda})$, it follows that $\nabla(\nabla \Xi(\cdot) \bar{\mu})(\bar{y})\left(g^{\prime}(\bar{x}) \xi\right)=\frac{1}{2} \nabla \Upsilon\left(g^{\prime}(\bar{x}) \xi\right)$. Thus, we complete the proof of this step.

Step 3: $\left\langle g^{\prime}(\bar{x}) \xi, v\right\rangle=\Upsilon\left(g^{\prime}(\bar{x}) \xi\right)$. Notice that $v=\frac{1}{2} \nabla \Upsilon\left(g^{\prime}(\bar{x}) \xi\right)+\eta$ with $\eta \in\left[\mathcal{C}_{\mathcal{K}}(\bar{y}, \bar{\lambda})\right]^{\circ}$ by Step 2. Also, from Step 2 and equation $(31), \nabla_{x x}^{2} L(\bar{x}, \bar{\lambda}) \xi+\nabla g(\bar{x}) v=0$, which means that $(\xi, \eta) \in \Sigma(\bar{x}, \bar{\lambda})$. Recall from Step 1 that $g^{\prime}(\bar{x}) \xi \in \mathcal{C}_{\mathcal{K}}(\bar{y}, \bar{\lambda})$. Then, $\left\langle g^{\prime}(\bar{x}) \xi, \eta\right\rangle \leq 0$. 
Together with the given assumption, we also have $\left\langle g^{\prime}(\bar{x}) \xi, \eta\right\rangle \geq 0$. Thus, $\left\langle\eta, g^{\prime}(\bar{x}) \xi\right\rangle=0$, and consequently $\left\langle g^{\prime}(\bar{x}) \xi, v\right\rangle=\left\langle g^{\prime}(\bar{x}) \xi, \frac{1}{2} \nabla \Upsilon\left(g^{\prime}(\bar{x}) \xi\right)\right\rangle=\Upsilon\left(g^{\prime}(\bar{x}) \xi\right)$.

So far, we have established that $g^{\prime}(\bar{x}) \xi \in \mathcal{C}_{\mathcal{K}}(\bar{y}, \bar{\lambda}), v-\frac{1}{2} \nabla \Upsilon\left(g^{\prime}(\bar{x}) \xi\right) \in\left[\mathcal{C}_{\mathcal{K}}(\bar{y}, \bar{\lambda})\right]^{\circ}$ and $\left\langle g^{\prime}(\bar{x}) \xi, v\right\rangle=\Upsilon\left(g^{\prime}(\bar{x}) \xi\right)$. By Lemma 2.3, this is equivalent to saying that

$$
g^{\prime}(\bar{x}) \xi-\Pi_{\mathcal{K}}^{\prime}\left(g(\bar{x})+\bar{\lambda} ; g^{\prime}(\bar{x}) \xi+v\right)=0 .
$$

Together with $\nabla_{x x}^{2} L(\bar{x}, \bar{\lambda}) \xi+\nabla g(\bar{x}) v=0$, it follows that $(\xi, v)$ satisfies the system (10). Since $\bar{\lambda}$ is noncritical, we obtain $\xi=0$, a contradiction to $\|\xi\|=1$.

From Proposition 2.2 and Lemma 3.1, the condition $\operatorname{ri}\left(\mathcal{N}_{\mathcal{K}}(g(\bar{x}))\right) \cap \mathcal{H}(\nabla f(\bar{x})) \neq \emptyset$ or equivalently $\operatorname{ri}\left(\mathcal{N}_{\mathcal{K}}(g(\bar{x}))\right) \cap \mathcal{M}(\bar{x}, 0,0) \neq \emptyset$ implies the calmness of $\mathcal{M}$ at $(\bar{x}, 0,0)$ for $\bar{\lambda} \in \mathcal{M}(\bar{x}, 0,0)$. While the following lemma states that if the system $g(x) \in \mathcal{K}$ is metrically subregular at $\bar{x}$, this condition also implies the closedness of $\nabla g(\bar{x})\left[\mathcal{C}_{\mathcal{K}}(g(\bar{x}), \bar{\lambda})\right]^{\circ}$.

Lemma 4.1 Let $(\bar{x}, \bar{\lambda})$ be a KKT point of the problem $(1)$ with $(a, b)=(0,0)$. Suppose that the multifunction $\mathcal{F}(\cdot):=g(\cdot)-\mathcal{K}$ is metrically subregular at $\bar{x}$ for the origin. If $\operatorname{ri}\left(\mathcal{N}_{\mathcal{K}}(g(\bar{x}))\right) \cap \mathcal{M}(\bar{x}, 0,0) \neq \emptyset$, then the radial cone $\mathcal{R}_{\mathcal{N}_{\Omega}(\bar{x})}(-\nabla f(\bar{x}))$ with $\Omega:=g^{-1}(\mathcal{K})$ is closed, which in turn implies the closedness of the set $\nabla g(\bar{x})\left[\mathcal{C}_{\mathcal{K}}(g(\bar{x}), \bar{\lambda})\right]^{\circ}$.

Proof: Notice that $\Omega$ is the feasible set of $(1)$ with $(a, b)=(0,0)$. Since $\mathcal{F}$ is metrically subregular at $\bar{x}$ for 0 , from [25, Corollary 2.1] we have $\mathcal{N}_{\Omega}(\bar{x})=\nabla g(\bar{x}) \mathcal{N}_{\mathcal{K}}(g(\bar{x}))$. From $\operatorname{ri}\left(\mathcal{N}_{\mathcal{K}}(g(\bar{x}))\right) \cap \mathcal{M}(\bar{x}, 0,0) \neq \emptyset$, there exists $\hat{\lambda} \in \operatorname{ri}\left(\mathcal{N}_{\mathcal{K}}(g(\bar{x}))\right)$ such that

$$
\nabla f(\bar{x})+\nabla g(\bar{x}) \widehat{\lambda}=0 .
$$

By the convexity of $\mathcal{N}_{\mathcal{K}}(g(\bar{x}))$, it follows that $\mathcal{N}_{\mathcal{K}}(g(\bar{x}))+\mathbb{R}_{+} \widehat{\lambda} \subseteq \mathcal{N}_{\mathcal{K}}(g(\bar{x}))$. Then, by following the arguments as for [15, Proposition 2.1], we have

$$
\begin{aligned}
\nabla g(\bar{x}) \mathcal{N}_{\mathcal{K}}(g(\bar{x}))+\llbracket \nabla g(\bar{x}) \widehat{\lambda} \rrbracket & =\nabla g(\bar{x})\left(\mathcal{N}_{\mathcal{K}}(g(\bar{x}))+\llbracket \widehat{\lambda} \rrbracket\right)=\nabla g(\bar{x})\left(\mathcal{N}_{\mathcal{K}}(g(\bar{x}))-\mathbb{R}_{+} \widehat{\lambda}\right) \\
& =\nabla g(\bar{x})\left[\mathbb{R}_{+}\left(\mathcal{N}_{\mathcal{K}}(g(\bar{x}))-\widehat{\lambda}\right)\right] \\
& =\operatorname{cl}\left\{\nabla g(\bar{x})\left[\mathbb{R}_{+}\left(\mathcal{N}_{\mathcal{K}}(g(\bar{x}))-\widehat{\lambda}\right)\right]\right\} \\
& =\operatorname{cl}\left\{\nabla g(\bar{x}) \mathcal{N}_{\mathcal{K}}(g(\bar{x}))+\llbracket \nabla g(\bar{x}) \widehat{\lambda} \rrbracket\right\}
\end{aligned}
$$

where the fourth equality is since $\mathbb{R}_{+}\left(\mathcal{N}_{\mathcal{K}}(g(\bar{x}))-\widehat{\lambda}\right)$ is a subspace parallel to the affine hull of $\mathcal{N}_{\mathcal{K}}(g(\bar{x}))$. Together with $\mathcal{N}_{\Omega}(\bar{x})=\nabla g(\bar{x}) \mathcal{N}_{\mathcal{K}}(g(\bar{x}))$ and (33), we obtain

$$
\begin{aligned}
\mathcal{R}_{\mathcal{N}_{\Omega}(\bar{x})}(-\nabla f(\bar{x})) & \left.=\nabla g(\bar{x}) \mathcal{N}_{\mathcal{K}}(g(\bar{x}))+\llbracket-\nabla f(\bar{x})\right]=\nabla g(\bar{x}) \mathcal{N}_{\mathcal{K}}(g(\bar{x}))+\llbracket \nabla g(\bar{x}) \widehat{\lambda} \rrbracket \\
& =\operatorname{cl}\left\{\nabla g(\bar{x}) \mathcal{N}_{\mathcal{K}}(g(\bar{x}))+\llbracket \nabla g(\bar{x}) \widehat{\lambda} \rrbracket\right\} \\
& =\operatorname{cl}\left\{\nabla g(\bar{x}) \mathcal{N}_{\mathcal{K}}(g(\bar{x}))+\llbracket-\nabla f(\bar{x}) \rrbracket\right\}=\operatorname{cl}\left(\mathcal{R}_{\mathcal{N}_{\Omega}(\bar{x})}(-\nabla f(\bar{x}))\right)
\end{aligned}
$$

where the first equality is due to [2, Exercise 2.62] and the closed convexity of $\mathcal{N}_{\Omega}(\bar{x})$. The last equality shows that the set $\mathcal{R}_{\mathcal{N}_{\Omega}(\bar{x})}(-\nabla f(\bar{x}))$ is closed. Notice that

$$
\begin{aligned}
\left.\nabla g(\bar{x}) \mathcal{N}_{\mathcal{K}}(g(\bar{x}))+\llbracket-\nabla f(\bar{x})\right) \rrbracket & =\nabla g(\bar{x})\left(\mathcal{N}_{\mathcal{K}}(g(\bar{x}))+\llbracket \bar{\lambda} \rrbracket\right) \subseteq \nabla g(\bar{x})\left[\mathcal{C}_{\mathcal{K}}(g(\bar{x}), \bar{\lambda})\right]^{\circ} \\
& \left.\subseteq \operatorname{cl}\left\{\nabla g(\bar{x}) \mathcal{N}_{\mathcal{K}}(g(\bar{x}))+\llbracket-\nabla f(\bar{x})\right) \rrbracket\right\} \\
& \left.=\nabla g(\bar{x}) \mathcal{N}_{\mathcal{K}}(g(\bar{x}))+\llbracket-\nabla f(\bar{x})\right) \rrbracket
\end{aligned}
$$


where the first equality is using the fact $\nabla f(\bar{x})+\nabla g(\bar{x}) \bar{\lambda}=0$, the first inclusion is by $\left[\mathcal{C}_{\mathcal{K}}(g(\bar{x}), \bar{\lambda})\right]^{\circ}=\operatorname{cl}\left\{\mathcal{N}_{\mathcal{K}}(g(\bar{x}))+\llbracket \bar{\lambda} \rrbracket\right\}$, and the second inclusion is due to [29, Theorem 6.6] and the convexity of $\mathcal{N}_{\mathcal{K}}(g(\bar{x}))$. This, along with the closedness of $\mathcal{R}_{\mathcal{N}_{\Omega}(\bar{x})}(-\nabla f(\bar{x}))$, shows that the set $\nabla g(\bar{x})\left[\mathcal{C}_{\mathcal{K}}(g(\bar{x}), \bar{\lambda})\right]^{\circ}$ is closed. The proof is completed.

Remark 4.1 (a) Notice that the condition $\operatorname{ri}\left(\mathcal{N}_{\mathcal{K}}(g(\bar{x}))\right) \cap \mathcal{M}(\bar{x}, 0,0) \neq \emptyset$ is much weaker than $\bar{\lambda} \in \operatorname{ri}\left(\mathcal{N}_{\mathcal{K}}(g(\bar{x}))\right)$ when the multiplier set $\mathcal{M}(\bar{x}, 0,0)$ is not a singleton.

(b) The metric subregularity of $\mathcal{F}(\cdot)=g(\cdot)-\mathcal{K}$ at $\bar{x}$ for the origin is a very weak constraint qualification (CQ), which is clearly implied by Robinson's CQ since the latter is equivalent to the metric regularity of $\mathcal{F}$ at $\bar{x}$ for the origin by [10]. For the research on the metric subregularity of the system $g(x) \in \mathcal{K}$, the reader may refer to [14, 17].

Now, by combining Lemma 4.1 with Proposition 4.2 and Theorem 3.2, we obtain the following sufficient characterization for the strong calmness of $\mathcal{S}_{\mathrm{KKT}}$.

Theorem 4.1 Let $(\bar{x}, \bar{\lambda})$ be a KKT point of $(1)$ with $(a, b)=(0,0)$ and $\mathcal{M}(\bar{x}, 0,0) \neq\{\bar{\lambda}\}$. Suppose the multifunction $\mathcal{F}(\cdot)=g(\cdot)-\mathcal{K}$ is metrically subregular at $\bar{x}$ for 0 . Define

$$
\widetilde{\Gamma}(\bar{x}, \bar{\lambda}):=\left\{(\xi, \zeta) \in \mathbb{X} \times \mathbb{Y}: g^{\prime}(\bar{x}) \xi \in \mathcal{C}_{\mathcal{K}}(g(\bar{x}), \bar{\lambda}), \nabla g(\bar{x}) \zeta \in \nabla g(\bar{x})\left(\mathcal{N}_{\mathcal{K}}(g(\bar{x}))+\llbracket \bar{\lambda} \rrbracket\right)\right\} .
$$

If $\left\langle g^{\prime}(\bar{x}) \xi, \zeta\right\rangle \geq 0$ for any $(\xi, \zeta) \in \Sigma(\bar{x}, \bar{\lambda}) \cap \widetilde{\Gamma}(\bar{x}, \bar{\lambda})$ and $\operatorname{ri}\left(\mathcal{N}_{\mathcal{K}}(g(\bar{x}))\right) \cap \mathcal{M}(\bar{x}, 0,0) \neq \emptyset$, the noncriticality of $\bar{\lambda}$ is enough to the strong calmness of $\mathcal{S}_{\mathrm{KKT}}$ at $(0,0)$ for $(\bar{x}, \bar{\lambda})$.

Proof: The proof is same as that of Proposition 4.2 except for the following fact

$$
\nabla g(\bar{x}) \eta \in \nabla g(\bar{x})\left[\mathcal{C}_{\mathcal{K}}(g(\bar{x}), \bar{\lambda})\right]^{\circ}=\nabla g(\bar{x})\left(\mathcal{N}_{\mathcal{K}}(g(\bar{x}))+\llbracket \bar{\lambda} \rrbracket\right)
$$

where $\eta$ is from Step 2 in the proof of Proposition 4.2, and the equality is by (34).

Remark 4.2 (a) For the case where $\mathcal{K}$ is the semidefinite positive cone, the condition that $\left\langle g^{\prime}(\bar{x}) \xi, \zeta\right\rangle \geq 0$ for any $(\xi, \zeta) \in \Sigma(\bar{x}, \bar{\lambda}) \cap \widetilde{\Gamma}(\bar{x}, \bar{\lambda})$ is implied by the condition (ii) of [35, Theorem 3.3], and the closedness condition of $\nabla g(\bar{x})\left[\mathcal{C}_{\mathcal{K}}(g(\bar{x}), \bar{\lambda})\right]^{\circ}$ there is removed. Thus, the result of Theorem 4.1 improves that of [35, Theorem 3.3] in this setting.

(b) When $\mathcal{M}(\bar{x}, 0,0)=\{\bar{\lambda}\}$, the strong calmness of $\mathcal{S}_{\mathrm{KKT}}$ at the origin for $(\bar{x}, \bar{\lambda})$ becomes its isolated calmness at the origin for $(\bar{x}, \bar{\lambda})$, which is equivalent to the calmness of $\mathcal{M}$ at $(\bar{x}, 0,0)$ for $\bar{\lambda}$ along with the noncriticality of the multiplier $\bar{\lambda}$; see Appendix.

Let $(\bar{x}, \bar{\lambda})$ be a KKT point of $(1)$ with $(a, b)=(0,0)$. Recall that the second-order sufficient condition holds at $\bar{x}$ w.r.t. the multiplier $\bar{\lambda}$ for $(1)$ with $(a, b)=(0,0)$ if

$$
\left\langle d, \nabla_{x x}^{2} L(\bar{x}, \bar{\lambda}) d\right\rangle-\sigma\left(\lambda, \mathcal{T}_{\mathcal{K}}^{2}\left(g(\bar{x}), g^{\prime}(\bar{x}) d\right)\right)>0 \quad \forall d \in \mathcal{C}(\bar{x}) \backslash\{0\} .
$$

Clearly, this SOSC is stronger than the SOSC at $\bar{x}$ stated in [2, Theorem $3.45 \& 3.137]$. By Proposition 2.1 and Lemma 2.3, a simple argument by contradiction shows that the SOSC at $\bar{x}$ w.r.t. $\bar{\lambda}$ implies the noncriticality of $\bar{\lambda}$. Then, under the conditions of Theorem 4.1, the SOSC at $\bar{x}$ w.r.t. $\bar{\lambda}$ is sufficient for the strong calmness of $\mathcal{S}_{\mathrm{KKT}}$. 


\section{Conclusions}

In this paper, for a class of canonically perturbed conic programming, we have provided two equivalent characterizations for the strong calmness of the KKT solution mapping, i.e., this property is equivalent to a local error bound for solutions of perturbed KKT system, as well as the pseudo-isolated calmness of the stationary point mapping along with the calmness of the multiplier set mapping. In addition, some weaker sufficient conditions than [7, Theorem 3.2] and [35, Theorem 3.3] for this property are also given in terms of the noncriticality of the Lagrange multiplier. The obtained results are crucial to achieve fast convergence of some algorithms such as the ALM for many important non-polyhedral convex conic optimization problems with degenerate solutions.

\section{References}

[1] J. P. Aubin, Contingent derivatives of set-valued maps and existence of solutions to nonlinear inclusions and differential inclusions, in Mathematical Analysis and Applications, Part A, edited by L. Nachbin, Advances in Mathematics: Supplementary Studies, 7A, pp. 160-232, Academic Press, New York,1980.

[2] J. F. Bonnans And A. Shapiro, Perturbation Analysis of Optimization Problems, Springer, New York. 2000.

[3] J. F. Bonnans, R. Cominetti And A. Shapiro, Sensitivity analysis of optimization problems under second order regular constraints, Mathematics of Operations Research, 23(1998): 806-831.

[4] J. F. Bonnans, R. Cominetti And A. Shapiro, Second-order optimality conditions based on parabolic second-order tangent sets, SIAM Journal on Optimization, 9(1999): 466-492.

[5] J. F. Bonnans and H. C. Ramírez, Perturbation analysis of second-order cone programming problems, Mathematical Programming, 104(2005): 205-227.

[6] H. H. Bauschke, J. M. Borwein And W. Li, Strong conical hull intersection property, bounded linear regularity, Jameson's property $(G)$, and error bounds in convex optimization, Mathematical Programming, 86(1999): 135-160.

[7] Y. Cui, D. F. Sun And K. C. ToH, On the asymptotic superlinear convergence of the augmented Lagrangian method for semidefinite programming with multiple solutions, arXiv: $1610.00875 \mathrm{v} 1,2016$.

[8] C. Ding, D. F. Sun And K. C. Toh, An introduction to a class of matrix cone programming, Mathematical Programming, 144(2014): 141-179.

[9] C. Ding, D. F. Sun And L. W. Zhang, Characterization of the robust isolated calmness for a class of conic programming problems, SIAM Journal on Optimization, 27(2017): 67-90. 
[10] A. L. Dontchev And R. T. Rockafellar, Implicit Functions and Solution Mappings-A View from Variational Analysis, Springer, 2009.

[11] D. Fernández And M. Solodov, Stabilized sequential quadratic programming for optimization and a stabilized Newton-type method for variational problems, Mathematical Programming, 125(2010): 47-73.

[12] A. FischeR, Local behavior of an iterative framework for generalized equations with nonisolated solutions, Mathematical Programming, 94(2002): 91-124.

[13] H. GFRERER, First order and second order characterizations of metric subregularity and calmness of constraint set mappings, SIAM Journal on Optimization, 21(2011): 1439-1474.

[14] H. GFrerer And J. V. OUtrata, On computation of generalized derivatives of the normal-cone mapping and their applications, Mathematics of Operations Research, 41(2016): 1535-1556.

[15] H. Gfrerer And B. S. Mordukhovich, Second-order variational analysis of parametric constraint and variational systems, arXiv:1711.07082v1, 2017.

[16] W. Hager And M. Gowda, Stability in the presence of degeneracy and error estimation, Mathematical Programming, 85(1999): 181-192.

[17] R. Henrion and J. V. Outrata, Calmness of constraint systems with applications, Mathematical Programming, 104(2005): 437-464.

[18] A. D. Ioffe And J. V. Outrata, On metric and calmness qualification conditions in subdifferential calculus, Set-Valued Analysis, 16(2008): 199-227.

[19] A. F. IzMailov, Solution sensitivity for Karush-Kuhn-Tucker systems with nonunique Lagrange multipliers, Optimization, 95(2010): 747-775.

[20] A. F. Izmailov And M. V. Solodov, Stabilized SQP revisited, Mathematical Programming, 133(2012): 93-120.

[21] A. F. Izmailov, A. S. Kurennoy And M. V. Solodov, A note on upper Lipschitz stability, error bounds, and critical multipliers for Lipschitz-continuous KKT systems, Mathematical Programming, 142(2013): 591-604.

[22] A. F. Izmailov, A. S. Kurennoy and M. V. Solodov, Local convergence of the method of multipliers for variational and optimization problems under the noncriticality assumption, Computational Optimization and Applications, 60(2015): 111-140.

[23] D. Klatte and B. Kummer, Constrained minima and Lipschitizan penalties in metric spaces, SIAM Journal on Optimization, 13(2002): 619-633.

[24] A. J. King And R. T. Rockafellar, Sensitivity analysis for nonsmooth generalized equations, Mathematical Programming, 55(1992): 193-212. 
[25] Y. L. LiU And S. H. PAN, Computation of graphical derivatives of normal cone maps to conic constraints without nondegeneracy and PDC, arXiv: 1709.05592v1, 2017.

[26] A. B. LEVY, Implicit multifunction theorems for the sensitivity analysis of variational conditions, Mathematical Programming, 74(1996): 333-350.

[27] B. S. MordukHovich, Maximum principle in problems of time optimal control with nonsmooth constraints, Journal of Applied Mathematics and Mechanics, 40(1976): 960-969.

[28] B. S. Mordukhovich, Variational Analysis and Generalized Differentiation I \& II, Springer-Verlag, Berlin Heidelberg, 2006.

[29] R. T. Rockafellar, Convex Analysis, Princeton University Press, 1970.

[30] S. M. Robinson, Some continuity properties of polyhedral multifunctions, Mathematical Programming Study, 14(1981): 206-214.

[31] S. M. Robinson, Generalized equations and their solutions, Part II: applications to nonlinear programming, Mathematical Programming Study, 10(1982): 200-221.

[32] R. T. Rockafellar and R. J-B. Wets, Variational Analysis, Springer, 1998.

[33] S. J. WRIGHT, Superlinear convergence of a stabilized SQP method to a degenerate Solution, Computational Optimization and Applications, 11(1998): 253-275.

[34] J. Wu, L. W. Zhang And Y. Zhang, Mathematical programs with semidefinite cone complementarity constraints: constraint qualifications and optimality conditions, Set-Valued and Variational Analysis, 22(2014): 155-187.

[35] T. Y. ZhANG AND L. W. ZHANG, Critical multipliers in semidefinite programming, arXiv: 1801.02218v1, 2018.

\section{Appendix}

Proposition 1 Let $(\bar{x}, \bar{\lambda})$ be a KKT point of the problem $(1)$ with $(a, b)=(0,0)$. Then, the isolated calmness of $\mathcal{X}_{\mathrm{KKT}}$ at the origin for $\bar{x}$ implies its pseudo-isolated calmness at the origin for $\bar{x}$. Also, the converse conclusion holds provided that Robinson's CQ holds at $\bar{x}$ for the problem $(1)$ with $(a, b)=(0,0)$ and $\mathcal{M}(\bar{x}, 0,0)=\{\bar{\lambda}\}$.

Proof: The proof of the first part is easy, and we here focus on the proof of the second part. Suppose that $\mathcal{X}_{\mathrm{KKT}}$ has the pseudo-isolated calmness at the origin for $\bar{x}$. If $\mathcal{X}_{\mathrm{KKT}}$ is not isolated calm at the origin for $\bar{x}$, then there exist the sequences $\left\{\left(a^{k}, b^{k}\right)\right\} \rightarrow 0$ and $\left\{x^{k}\right\} \rightarrow \bar{x}$ with $x^{k} \in \mathcal{X}_{\mathrm{KKT}}\left(a^{k}, b^{k}\right)$ for each $k \in \mathbb{N}$ such that $\frac{\left\|\left(a^{k}, b^{k}\right)\right\|}{\left\|x^{k}-\bar{x}\right\|} \rightarrow 0$ as $k \rightarrow \infty$. Since $x^{k} \in \mathcal{X}_{\mathrm{KKT}}\left(a^{k}, b^{k}\right)$, for each $k \in \mathbb{N}$ there exists $\lambda^{k} \in \mathcal{M}\left(x^{k}, a^{k}, b^{k}\right)$ such that $\left(x^{k}, \lambda^{k}\right) \in \mathcal{S}_{\mathrm{KKT}}\left(a^{k}, b^{k}\right)$. Together with the pseudo-isolated calmness of $\mathcal{X}_{\mathrm{KKT}}$ at the 
origin for $\bar{x}$, there exists $\epsilon_{0}>0$ such that for all sufficiently large $k,\left\|\lambda^{k}-\bar{\lambda}\right\| \geq \epsilon_{0}$. On the other hand, since Robinson's CQ holds at $\bar{x}$ for $(1)$ with $(a, b)=(0,0)$, the multifunction $\mathcal{M}$ is locally bounded at $(\bar{x}, 0,0)$ in the sense of [32, Definition 5.14] (see also the proof of [31, Theorem 3.2]). Thus, the sequence $\left\{\lambda^{k}\right\}$ is bounded. Without loss of generality, assume that $\lambda^{k} \rightarrow \hat{\lambda}$. Since $\left(x^{k}, \lambda^{k}\right) \in \mathcal{S}_{\mathrm{KKT}}\left(a^{k}, b^{k}\right)$, for each $k \in \mathbb{N}$ it holds that

$$
\nabla f\left(x^{k}\right)+\nabla g\left(x^{k}\right) \lambda^{k}=a^{k} \text { and } g\left(x^{k}\right)-\Pi_{\mathcal{K}}\left(g\left(x^{k}\right)-b^{k}+\lambda^{k}\right)=b^{k}
$$

Taking the limit $k \rightarrow \infty$ on the last two equalities, we obtain $\hat{\lambda} \in \mathcal{M}(\bar{x}, 0,0)=\{\bar{\lambda}\}$. This yields a contradiction to the result that $\left\|\lambda^{k}-\bar{\lambda}\right\| \geq \epsilon_{0}$ for all sufficiently large $k$.

Proposition 2 Let $(\bar{x}, \bar{\lambda})$ be a KKT point of $(1)$ with $(a, b)=(0,0)$. The multifunction $\mathcal{S}_{\mathrm{KKT}}$ is isolated calm at $(0,0)$ for $(\bar{x}, \bar{\lambda})$ if and only if $\mathcal{M}$ is isolated calm at $(\bar{x}, 0,0)$ for $\bar{\lambda}$ and the multiplier $\bar{\lambda}$ is noncritical for $(1)$ with $(a, b)=(0,0)$.

Proof: By [9, Lemma $18 \& 19], \mathcal{S}_{\mathrm{KKT}}$ is isolated calm at the origin for $(\bar{x}, \bar{\lambda})$ iff system

$$
\left\{\begin{array}{l}
\nabla_{x x}^{2} L(\bar{x}, \bar{\lambda}) u+\nabla g(\bar{x}) v=0 \\
g^{\prime}(\bar{x}) u-\Pi_{\mathcal{K}}^{\prime}\left(g(\bar{x})+\bar{\lambda} ; g^{\prime}(\bar{x}) u+v\right)=0
\end{array}\right.
$$

has only the trivial solution $(u, v)=(0,0)$. From the proof of Lemma 3.1, it is easy to obtain that $\mathcal{M}$ is isolated calm at $(\bar{x}, 0,0)$ for $\bar{\lambda}$ iff $\mathcal{G}_{\bar{x}}$ is isolated calm at $(\nabla f(\bar{x}), \nabla g(\bar{x}))$ for $\bar{\lambda}$. Thus, together with Proposition 2.1, it suffices to argue that the isolated calmness of $\mathcal{G}_{\bar{x}}$ at $(\nabla f(\bar{x}), \nabla g(\bar{x}))$ for $\bar{\lambda}$ is equivalent to saying $\Delta \lambda=0$ is the unique solution of

$$
\left\{\begin{array}{l}
\nabla g(\bar{x}) \Delta \lambda=0 \\
\Pi_{\mathcal{K}}^{\prime}(g(\bar{x})+\bar{\lambda} ; \Delta \lambda)=0 .
\end{array}\right.
$$

Indeed, it is not hard to check that gph $\mathcal{G}_{\bar{x}}=\mathcal{A}^{-1}\left(\operatorname{gph} \Phi^{-1}\right)$ with $\Phi$ and $\mathcal{A}$ defined by

$$
\Phi(\lambda):=\left(\begin{array}{c}
\nabla g(\bar{x}) \lambda \\
-\Pi_{\mathcal{K}}(\lambda)
\end{array}\right) \text { and } \mathcal{A}(\eta, y, \lambda):=\left(\begin{array}{c}
-\eta+\nabla g(\bar{x}) y \\
-y \\
\lambda+y
\end{array}\right)
$$

Write $\bar{\eta}=\nabla f(\bar{x})$ and $\bar{y}=g(\bar{x})$. Since $\mathcal{A}$ is bijective, by [32, Exercise 6.7] we have

$$
\mathcal{T}_{\text {gph } \mathcal{G}_{\bar{x}}}(\bar{\eta}, \bar{y}, \bar{\lambda})=\mathcal{A}^{-1}\left[\mathcal{T}_{\operatorname{gph} \Phi^{-1}}(-\bar{\eta}+\nabla g(\bar{x}) \bar{y},-\bar{y}, \bar{\lambda}+\bar{y})\right] .
$$

This, along with the definition of graphical derivative, gives the following equivalence:

$$
\Delta \lambda \in D \mathcal{G}_{\bar{x}}((\bar{\eta}, \bar{y}) \mid \bar{\lambda})(0,0) \Longleftrightarrow \Delta \lambda \in D \Phi^{-1}((-\bar{\eta}+\nabla g(\bar{x}) \bar{y},-\bar{y}) \mid \bar{y}+\bar{\lambda})(0,0) .
$$

Then, $D \mathcal{G}_{\bar{x}}((\bar{\eta}, \bar{y}) \mid \bar{\lambda})(0,0)=\{0\}$ if and only if $D \Phi^{-1}((-\bar{\eta}+\nabla g(\bar{x}) \bar{y},-\bar{y}) \mid \bar{y}+\bar{\lambda})(0,0)=\{0\}$. By Lemma $2.1, \mathcal{G}_{\bar{x}}$ is isolated calm at $(\bar{\eta}, \bar{y})$ for $\bar{\lambda}$ if and only if $\Phi^{-1}$ is isolated calm at $(-\bar{\eta}+\nabla g(\bar{x}) \bar{y},-\bar{y})$ for $\bar{y}+\bar{\lambda}$. In addition, by virtue of the Lipschitz continuity and the directional differentiability of $\Phi$, the following equivalence holds:

$$
\begin{aligned}
\Phi^{\prime}(\bar{y}+\bar{\lambda} ; \Delta \lambda)=\left(\begin{array}{c}
\Delta \eta \\
\Delta y
\end{array}\right) & \Longleftrightarrow(\Delta \eta, \Delta y) \in D \Phi(\bar{y}+\bar{\lambda} \mid(-\bar{\eta}+\nabla g(\bar{x}) \bar{y},-\bar{y}))(\Delta \lambda) \\
& \Longleftrightarrow \Delta \lambda \in D \Phi^{-1}((-\bar{\eta}+\nabla g(\bar{x}) \bar{y},-\bar{y}) \mid \bar{y}+\bar{\lambda})(\Delta \eta, \Delta y) .
\end{aligned}
$$

Thus, using Lemma 2.1 again delivers the desired statement. 